Controversies and prospects about microglia in maternal immune activation models for neurodevelopmental disorders.

Peer-reviewed author version

SMOLDERS, Silke; Notter, Tina; SMOLDERS, Sophie; RIGO, Jean-Michel \& BRONE, Bert (2018) Controversies and prospects about microglia in maternal immune activation models for neurodevelopmental disorders.. In: BRAIN BEHAVIOR AND IMMUNITY, 73, p. 51-65.

DOI: 10.1016/j.bbi.2018.06.001

Handle: http://hdl.handle.net/1942/28236 
1 Controversies and prospects about microglia in maternal immune activation

2

3 Silke Smolders ${ }^{1}$, Tina Notter ${ }^{2}$, Sophie MT Smolders ${ }^{3}$, Jean-Michel Rigo ${ }^{4}$, Bert Brône ${ }^{4}$

41 BIOMED - Uhasselt, Hasselt, Belgium models for neurodevelopmental disorders.

Laboratory of Neuronal Differentiation, VIB Center for the Biology of Disease, Leuven and Center for Human Genetics, KU Leuven Leuven, Belgium

2 Institute of Pharmacology and Toxicology, University of Zurich-Vetsuisse, Zurich, Switzerland Neuroscience Center Zurich, University of Zurich and ETH Zurich, Zurich, Switzerland.

3 BIOMED - Uhasselt, Hasselt, Belgium

INSERM, UMR S 1130, Université Pierre et Marie Curie Paris, France; CNRS, UMR 8246,

Université Pierre et Marie Curie Paris, France; UM 119 NPS, Université Pierre et Marie Curie Paris, France

4 BIOMED - Uhasselt, Hasselt, Belgium

\section{Mailing addresses:}

silke.smolders@kuleuven.vib.be, tina.notter@uzh.ch,sophie.smolders@uhasselt.be, jeanmichel.rigo@uhasselt.be, bert.brone@uhasselt.be

Individual, address, and telephone number to whom correspondence concerning manuscript should be sent:

Bert Brône

Martelarenlaan 42, 3500 Hasselt, Belgium

+3211269237ｂert.brone@uhasselt.be

Article type: Review

Declarations of interest: none 


\section{Abstract}

2 Activation of the maternal immune system during pregnancy is a well-established risk factor for

3 neuropsychiatric disease in the offspring, yet, the underlying mechanisms leading to altered brain

4 function remain largely undefined. Microglia, the resident immune cells of the brain, are key to

5 adequate development of the central nervous system (CNS), and are prime candidates to mediate

6 maternal immune activation (MIA)-induced brain abnormalities. As such, the effects of MIA on the

7 immunological phenotype of microglia has been widely investigated. However, contradicting results

8 due to differences in read-out and methodological approaches impede final conclusions on MIA-

9 induced microglial alterations. The aim of this review is to critically discuss the evidence for an

10 activated microglial phenotype upon MIA. 


\section{1. Introduction}

2 Maternal immune activation during pregnancy has been recognized to increase the risk for

3 neurodevelopmental psychiatric disorders such as schizophrenia, Autism Spectrum Disorders (ASD)

4 and bipolar disorder. Numerous epidemiological studies identified a number of inflammation-inducing

5 agents to be associated with psychiatric illnesses including prenatal exposure to viruses such as rubella,

6 herpes simplex, measles and polio (Torrey et al. 1988, Suvisaari et al. 1999, Brown et al. 2001, Buka et

7 al. 2001), various bacterial pathogens (Sorensen et al. 2009), and protozoan parasite such as

8 Taxoplasma gondii (Mortensen et al. 2007). Animal models of maternal immune activation (MIA) have

9 further established causality by showing that immune activation during pregnancy can perturb processes of early neurodevelopment in the offspring and change developmental trajectories. These MIA models precipitate into long-term behavioral and neuronal deficits relevant for psychiatric illnesses such as schizophrenia, ASD and related disorders (as reviewed in a.o. (Boksa 2010, Meyer 2014, Solek et al. 2017)).

In the MIA model, the maternal immune system can be stimulated by a variety of immune-activating agents including virus ((Fatemi et al. 1998) and others), lipopolysaccharide (LPS) ((Borrell et al. 2002) and others) and polyriboinosinic-polyribocytidilic acid (Poly(I:C)) ((Meyer et al. 2005) and others) resulting in viral, bacterial-like and viral-like immune responses respectively. Intriguingly, the structural and functional abnormalities in offspring exposed to MIA seem not to be dependent on the immuneactivating agents per se but critically depend on the general maternal and fetal immune response (Smith et al. 2007, Choi et al. 2016). Specific cytokines including interleukin (IL) 6 and IL17a have been identified as key players in mediating the disruption of neurodevelopmental processes in the MIA models (Smith et al. 2007, Choi et al. 2016). Moreover, the importance of the maternal gut microbiota in the response to MIA has recently been eluded by Kim et al. 2017, showing that treatment with the broad spectrum antibiotic vancomycin prior to MIA was able to prevent the emergence of behavioral 
which prenatal immune activation perturbs early brain development and leads to behavioral and neuronal deficits remain largely unknown. In recent years, there has been an increasing interest in the functional contribution of the neuro-immune system, and in particular aberrant microglia function, in the pathophysiological processes underlying neurodevelopmental disorders such as schizophrenia and ASD.

Microglia are the resident immune cells of the central nervous system (CNS). They are derived from primitive yolk-sac macrophages that invade the brain during early development (Ginhoux et al. 2010). In the adult brain, microglia actively scan the CNS parenchyma for potential changes in brain homeostasis or harmful insults by protruding and retracting their processes (Nimmerjahn et al. 2005). Once microglia sense changes in CNS integrity, they react accordingly. The classical response involves a process that is often referred to as "microglia activation" and includes changes in the expression profile of distinct markers of microglia, increased production and secretion of pro- and antiinflammatory cytokines and chemokines, and a shift in the morphological appearance from ramified to a more amoeboid morphology (Ransohoff and Cardona 2010). This classical immune-related activation refers to microglia responses towards harmful stimuli such as damage or invading pathogens. Over the past decade, however, the functional role of microglia has been extended to nonimmunological functions including the regulation of neurogenesis, myelination and synaptic remodeling (Bilimoria and Stevens 2015, Salter and Stevens 2017). As a consequence, the sustained activity of microglial protrusions might also reflect a continuous contribution in the ongoing remodeling of the brain instead of simply scanning the brain (Mosser et al. 2017). Microglia have thus been attributed a key role in neurodevelopmental processes, including the establishment of proper neuronal connectivity and brain homeostasis (as extensively reviewed in (Arnold and Betsholtz 2013, Bilimoria and Stevens 2015, Wu et al. 2015, Frost and Schafer 2016, Reemst et al. 2016, Mosser et al. 2017, Paolicelli and Ferretti 2017)). Given these neuro-modulatory functions, abnormal microglia activity may thus alter neuronal development and thereby contribute to the behavioral and neuronal deficits observed in neurodevelopmental disorders. 
Consequently, it has been proposed that prenatal immune activation could act directly on microglia and thereby alter their function in the developing brain, resulting in changing the developmental trajectories and ultimately leading to the behavioral and neuronal deficits detected in MIA models (Knuesel et al. 2014, Paolicelli and Ferretti 2017). In support of this hypothesis is the identification of signs of central inflammatory processes and microglia anomalies in post-mortem brains of a subset of schizophrenic and ASD patients (Vargas et al. 2005, Morgan et al. 2010, Trepanier et al. 2016, van Kesteren et al. 2017). Besides this, there have been numerous studies that investigated microglia phenotypes in the MIA model in order to associate microglia activity with brain and behavioral abnormalities (Arsenault et al. 2014, Bilbo et al. 2017, Paolicelli and Ferretti 2017). However, the reports are inconsistent with some detecting microglia with an altered phenotype (e.g. (Esslinger et al. 2016, Gumusoglu et al. 2017)) whereas others detect no changes in microglia biology in offspring prenatally exposed to MIA (e.g. (Giovanoli et al. 2015, Smolders et al. 2015)). It is one of the main aims of this review to thoroughly discuss the controversies in the field and critically revise possible factors that could contribute to the controversial findings regarding microglia alterations in the MIA model. Among these factors are those associated to the commonly used microglial activation read-outs (such as the age, sex and brain region analyzed) as well as MIA-model associated factors (such as the immune stimulant used and the age of MIA induction). We aim to conclude with suggestions/guidelines to improve the consistency in the field of MIA-microglia research.

\section{Methods}

The publications discussed in paragraph 3 were retrieved by searching the Title and/or Abstract field in pubmed using the following search terms: 'maternal immune activation', 'prenatal immune activation', 'maternal immune stimulation', 'prenatal immune stimulation', 'maternal immune challenge', 'prenatal immune challenge', 'prenatal infection', 'prenatal viral infection', 'prenatal bacterial infection', ' maternal viral infection', 'maternal bacterial infection', 'maternal infection' or 
'maternal inflammation'. Every publication found by these search terms was opened and inspected for analysis on microglia. Whilst the MIA model has been extended to pigs (Antonson et al. 2017) and (non)-human primates (Careaga et al. 2017) we will only discuss and revise studies that systemically administered immune agents such as LPS or Poly(I:C) or specific cytokines (excluding intrauterine administration) in rodents including mice and rats. Although early postnatal injections (either through direct infection of the neonate or through infection of the lactating dam) of Poly(I:C) have been used to mimic late-gestation infection in humans (Ribeiro et al. 2013, Arad et al. 2017, Monte et al. 2017), we will only consider studies inducing MIA at prenatal stages in order to include the effects of the maternal immune system and contribution of the placenta.

\section{Microglial reaction to maternal infection}

The findings on MIA-induced alterations of microglia activity in rodents are divergent. There are reports that describe alterations in the microglia activity state that point towards a pro-inflammatory phenotype. These include increased microglia density (here defined as amount of cells $/ \mathrm{mm}^{2}$ ) (e.g. (Juckel et al. 2011, Manitz et al. 2013, Li et al. 2014, Zhu et al. 2014)), increased expression of microglia "activation" markers (e.g. Major histocompatibility complex II (MHCII), Cluster of differentiation (CD) 68 or CD54 (Borrell et al. 2002, Van den Eynde et al. 2014, Esslinger et al. 2016)), and changes in the morphological appearance (e.g. altered microglial branches (Juckel et al. 2011, Van den Eynde et al. 2014, Corradini et al. 2017)). On the other hand, there are multiple studies where no obvious changes in microglia activity were detected (e.g. (Garay et al. 2013, Giovanoli et al. 2015, Giovanoli et al. 2016)). Multiple reasons may underlie these contradictory results and should be considered when comparing the outcome of different studies on "microglia activation".

Firstly, "microglial activation" can be assessed in several ways, some of which are more sensitive than others. The most common methods used in MIA models include the estimation of the number/density 
of microglia by means of stereology (e.g. (Mattei et al. 2014, Giovanoli et al. 2015, Hadar et al. 2016)), and the morphological appearance of microglia (e.g. (Giovanoli et al. 2015, Hsueh et al. 2017, Notter et al. 2017)). Another common approach to measure microglia activity is the assessment of the expression - usually by means of immunohistochemistry or biochemistry - of microglia activation markers (such as CD68 (Esslinger et al. 2016, Manitz et al. 2016, Notter et al. 2017) and Translocator protein (TSPO) (Mattei et al. 2017, Notter et al. 2017). To identify or isolate microglia from the brain, samples obtained from non-transgenic animals are stained with markers that are highly expressed by microglia (such as ionized calcium-binding adapter molecule 1 (Iba1), CD11b or CD68) or samples are obtained from transgenic animals where a reporter gene is expressed under the CX3C chemokine receptor 1 (CX3CR1) promotor. Although these molecular markers for microglia are often considered to be microglia-specific, they are also expressed by other members of the mononuclear phagocyte system such as monocytes and macrophages. Therefore, many groups have recently searched for a molecular marker that allows to distinguish between microglia and other immune cells in the brain. As such, the expression of the transcriptional repressor Spalt Like Transcription Factor 1 (Sall1) was recently shown to be largely restricted to microglia in the adult brain, although some cells in the liver, kidney and heart were also found to express this marker (Buttgereit et al. 2016). Similarly, Transmembrane protein 119 (Tmem119) was identified as a microglia-specific marker in the human and mouse CNS (Bennett et al. 2016). Although these markers will be of great value to distinguish microglia in the adult brain, their use in embryonic contexts should be further investigated.

Besides the methodology and readouts used to assess microglia activation state, the brain region investigated and time point/developmental stage when microglia are analyzed differ among the different studies, which makes direct comparisons merely impossible. In addition to these analytical issues, there are numerous other factors to be considered including the immune stimulant used, gestational window of immune activation, frequency of immune challenges, and route of administration that could all contribute to the observed inconsistencies in microglia phenotypes in the MIA model. Moreover, factors that are not directly related to the method of MIA-induction or microglia 
activation assessment could influence the outcome of MIA studies, including housing conditions, food pellet composition and specific mouse strain (Babri et al. 2014, Li et al. 2015, Buschert et al. 2016, Weiser et al. 2016).

The vast majority of studies have analyzed MIA-induced alterations of microglial activity during postnatal stages while only a few studies have focused on microglial changes during prenatal developmental stages. In the first section, we focus on findings derived from offspring in postnatal stages subdivided according to the specific read-out used to assess microglia activation (number/density, morphology, expression of microglia activity dependent genes and proteins) in order to compare different MIA-microglia studies easier. However, as will be discussed later in this review, even by dividing studies in this manner, many other variables make direct comparison merely impossible. In the subsequent section, findings obtained during prenatal development are discussed.

\subsection{Readout Postnatal Stage}

\section{Microglia Number/Density}

Upon detection of an insult, microglia are known to migrate to the site of injury/infection and/or proliferate, a phenomenon called microgliosis (Streit et al. 1999). "Microglial activation" is thus often measured in relation to its number/density in specific brain regions of interest. Despite seemingly straightforward, the density of microglia can vary depending on the age and sex of the animal, as well as the brain region investigated (Schwarz et al. 2012) (as will be discussed in section 4). Moreover, studies differ with regard to the marker used to identify microglia (Iba1 or CD11b) and therefore, in the tables below, the specific markers are mentioned. In addition, the variety in techniques to determine microglia density (fluorescent vs chromogenic staining and software) might lead to variability in results between different studies.

A total of 21 studies were included in this review assessing microglia density in different brain regions of rat and mice offspring exposed to MIA (Table 1). Out of these, 19 studies measured microglia in 
1 adult offspring (age > P60). A minority of these studies (only 5) reported an increased density of

2 microglia in several brain regions while the vast majority (14 studies) did not detect a change in

3 microglial density. Similar to the findings in adult brain, measurements in younger, adolescent

4 offspring provides more evidence for unchanged microglial densities (4 studies) in comparison to

5 increased density (only 2 studies).

6 These results suggest that MIA only sporadically induces microgliosis in certain brain regions. The

7 biological function of increased numbers of microglia in specific brain regions of MIA exposed offspring

8 and its consequences on brain formation and maturation has yet to be identified.

9 Table 1: Microglia number/density in postnatal MIA offspring

\section{Microglia Number/Density}

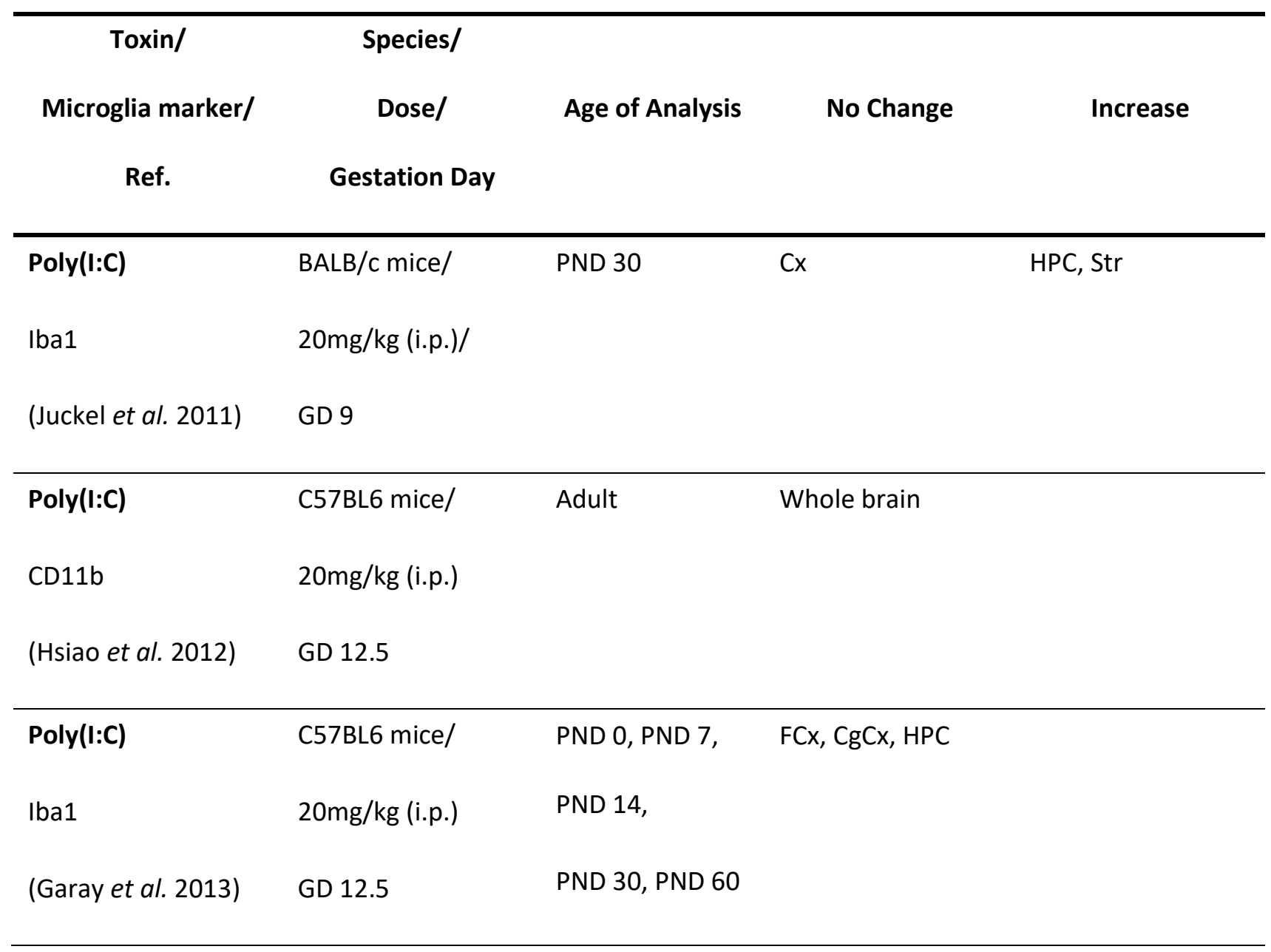




\begin{tabular}{|c|c|c|c|c|}
\hline Poly(I:C) & BALB/c mice/ & PND 10 & Str, FaCx, HPC, & \\
\hline Iba1 & $20 \mathrm{mg} / \mathrm{kg}$ (i.p.) & & VCx, MCx & \\
\hline \multirow[t]{2}{*}{ (Manitz et al. 2013) } & GD 9.5 & PND 30 & Str, MCx & $\mathrm{FaCx}, \mathrm{HPC}$ \\
\hline & & PND 100 & $\mathrm{HPC}, \mathrm{VCx}, \mathrm{MCx}$ & Str, $\mathrm{FaCx}$ \\
\hline Poly(I:C) & C57BL6 mice/ & PND 85 & $\mathrm{mPfCx}, \mathrm{Str}$ & \\
\hline Iba1 & $5 \mathrm{mg} / \mathrm{kg}$ (i.v.) & & & \\
\hline (Willi et al. 2013) & GD 9 & & & \\
\hline Poly(I:C) & C57BL6 mice/ & PND 2 & & HPC \\
\hline Iba1 & $20 \mathrm{mg} / \mathrm{kg}$ (i.p.) & PND 80 & $\mathrm{HPC}$ & \\
\hline (Li et al. 2014) & GD 9.5 & & & \\
\hline Poly(I:C) & C57BL6 mice/ & PND 62 & & Th, Cx, HPC \\
\hline Iba1 & $20 \mathrm{mg} / \mathrm{kg}$ (i.p.) & & & \\
\hline (Zhu et al. 2014) & GD 9 & & & \\
\hline Poly(I:C) & C57BL6 mice/ & PND 28, PND & $\mathrm{HPC}$ & \\
\hline Iba1 & $5 \mathrm{mg} / \mathrm{kg}$ (i.v.) & 140, PND 600 & & \\
\hline (Giovanoli et al. & GD 17 & & & \\
\hline \multicolumn{5}{|l|}{ 2015) } \\
\hline Poly(I:C) & CD-1 mice/ & PND 172 & & \\
\hline Iba1 & $20 \mathrm{mg} / \mathrm{kg}$ (i.p.) & Standard & $\mathrm{CX}, \mathrm{HPC}$ & \\
\hline (Buschert et al. & GD 9.5 & housing & & \\
\hline 2016) & & Enriched & $\mathrm{Cx}, \mathrm{HPC}$ & \\
\hline & & housing & & \\
\hline
\end{tabular}




\begin{tabular}{|c|c|c|c|c|}
\hline Poly(I:C) & C57BL6 mice/ & PND 35, PND 84 & HPC & \\
\hline Iba1 & $5 \mathrm{mg} / \mathrm{kg}$ (i.v.) & & & \\
\hline (Giovanoli et al. & GD 9 & & & \\
\hline \multicolumn{5}{|l|}{ 2016) } \\
\hline Poly(I:C) & C57BL6 mice/ & PND 90 & $C x$ & \\
\hline Iba1 & $2 \mathrm{mg} / \mathrm{kg}$ (i.p.) & & & \\
\hline (Corradini et al. & GD 9 & & & \\
\hline \multicolumn{5}{|l|}{ 2017) } \\
\hline Poly(I:C) & C57BL6 mice/ & PND 90 & $\mathrm{mPfCx}$ & \\
\hline Iba1 & $5 \mathrm{mg} / \mathrm{kg}$ (i.v.) & & & \\
\hline (Notter et al. 2017) & GD 9 & & & \\
\hline Poly(I:C) & C57BL6 mice/ & PND 80 & $\mathrm{PfCx}, \mathrm{Cb}, \mathrm{HPC}$ & \\
\hline Iba1 & $5 \mathrm{mg} / \mathrm{kg}$ (i.p.) & & & \\
\hline (Hui et al. 2018) & GD 9.5 & & & \\
\hline Poly(I:C) & Lewis rat/ & PND 9 & & Forebrain \\
\hline Iba1 & $200 \mu \mathrm{g} / \mathrm{kg}$ & & & White matter \\
\hline \multirow[t]{3}{*}{ (Girard et al. 2010) } & (i.p.)/every $12 \mathrm{~h}$ & & & \\
\hline & starting from & & & \\
\hline & GD 17.5 until birth & & & \\
\hline Poly(I:C) & Wistar rat/ & PND 128 & Str, Cb, HPC, PfCx & NAc \\
\hline Iba1 & $4 \mathrm{mg} / \mathrm{kg}$ (i.v.) & & & \\
\hline (Mattei et al. 2014) & GD 15.5 & & & \\
\hline
\end{tabular}




\begin{tabular}{|c|c|c|c|c|}
\hline Poly(I:C) & Wistar-Hannover & PND 90 & FtCx, CC, HPC, & \\
\hline CD11b/Iba1 & rat/ & & Th, Str, Pons & \\
\hline (Missault et al. & 4 mg/kg (i.v.) & & & \\
\hline 2014) & GD 15.5 & & & \\
\hline Poly(I:C) & Sprague Dawley & PND 180 & Th, Cx, HPC, Str, & Pons, CC \\
\hline CD11b & rat/ & & & \\
\hline (Van den Eynde et & 4 mg/kg (i.v.) & & & \\
\hline al. 2014) & GD 15.5 & & & \\
\hline Poly(I:C) & Wistar rat/ & PND 130 & $\mathrm{PfCx}$ & NAc, HPC \\
\hline Iba1 & 4 mg/kg (i.v.) & & & \\
\hline (Hadar et al. 2016) & GD15.5 & & & \\
\hline Poly(I:C) & Long-Evans rat/ & PND 7, PND 21, & $\mathrm{MpCx}, \mathrm{FaCx}$ & \\
\hline Iba1 & 4 mg/kg (i.v.) & PND 35, PND 90 & $\mathrm{PaCx}, \mathrm{Am}$ & \\
\hline (Paylor et al. 2016) & GD 15.5 & & & \\
\hline LPS & C57BL6 mice/ & PND 56 & $C x$ & \\
\hline Iba1 & $25-50 \mu / \mathrm{kg}$ (i.v.) & & & \\
\hline (Hsueh et al. 2017) & GD $15-17$ & & & \\
\hline LPS & Sprague Dawley & PND 70 & HPC & \\
\hline lba1 & rat/ & & & \\
\hline \multirow[t]{2}{*}{ (Mouihate 2016) } & $100 \mu \mathrm{g} / \mathrm{kg}$ (i.p.) & & & \\
\hline & GD 15.5 & & & \\
\hline
\end{tabular}


HPC: Hippocampus, NAc: Nucleus accumbens, Str: Striatum, Th: Thalamus, Cx: Cortex, CgCx: Cingulate Cortex, VCx: Visual Cortex, MCx: Motor cortex, FaCx: Frontal association Cortex, FCx: Frontal Cortex, FtCx: Frontotemporal Cortex, (m)PfCx: (medial) Prefrontal Cortex, MpCx: Medial Prelimbic Cortex, PaCx: Primary Auditory Cortex, CC: Corpus Callosum, Cb: Cerebellum, Am: Amygdala

\section{Microglia Morphology}

A second parameter often used to assess "microglia activation" is the cell's morphology. The fact that pathological insults can cause microglia to adopt a more amoeboid shape has led to the assumption that ramified microglia represent a "resting" state while amoeboid forms were interpreted as "activated" state (Hanisch and Kettenmann 2007). Single cell transcriptome studies in microglia, however, revealed that microglia activation states cannot be simply classified into "resting" and "active" states but rather represents a continuum at which ramified microglia represent an active form as well (Crotti and Ransohoff 2016). Hence, equating microglia morphology with microglia activation could therefore result in false negative results and misleading conclusions. Indeed, changes in microglia morphology represent an adequate response towards a specific insult but should not be taken as a general sign of microglia activation (Harry and Kraft 2012, Streit et al. 2014). On top of that, during brain development, the morphology of microglia is changing gradually from amoeboid to ramified and the age of the animals at which microglial morphology is studied might contribute to contradictory results. Conversely, the fact that microglia are ramified does not necessarily mean they are not responsive to an inflammatory stimulus on the transcriptional level: it was shown that 2-12 hours after a systemic inflammatory stimulus, transcriptional changes occurred in microglia while changes in morphology were only detected after 24-48 hours (Norden et al. 2016).

Nevertheless, multiple MIA studies have taken morphological measures to assess microglia activity (Table 2). Similar to the microglia density analyses, the assessment of microglial morphology in MIA has resulted in contradictory findings. The findings of these studies vary from decreased numbers of 
1 processes (Juckel et al. 2011, Van den Eynde et al. 2014, Corradini et al. 2017, O'Loughlin et al. 2017,

2 Hui et al. 2018) - indicative of the amoeboid state - to increased ramification (Gumusoglu et al. 2017).

3 In comparison to these 6 studies, however, the 7 other studies presented in this review detected no

4 changes in the microglia morphology of MIA exposed adult offspring (Garay et al. 2013, Missault et al.

5 2014, Giovanoli et al. 2015, Giovanoli et al. 2016, Mouihate 2016, Hsueh et al. 2017, Notter et al. 2017).

6 Table 2: Microglia morphology in postnatal MIA offspring

\section{Microglia Morphology}

\begin{tabular}{|c|c|c|c|c|}
\hline $\begin{array}{c}\text { Toxin/ } \\
\text { Microglia marker/ } \\
\text { Ref. }\end{array}$ & $\begin{array}{c}\text { Species/ } \\
\text { Dose/ } \\
\text { Gestation Day }\end{array}$ & $\begin{array}{l}\text { Age of } \\
\text { Analysis }\end{array}$ & Regions analyzed & Findings \\
\hline Poly(I:C) & BALB/c mice/ & & & Decreased density and \\
\hline Iba1 & 20mg/kg (i.p.)/ & PND 30 & $\mathrm{HPC}, \mathrm{Cx}, \mathrm{Str}$ & amount of branches and \\
\hline (Juckel et al. 2011) & GD 9 & & & processes \\
\hline Poly(I:C) & C57BL6 mice/ & PND 0, & & \\
\hline Iba1 & 20mg/kg (i.p.) & PND 7, & & \\
\hline \multirow[t]{3}{*}{ (Garay et al. 2013) } & GD 12.5 & PND 14, & $\mathrm{FtCx}, \mathrm{HPC}, \mathrm{CgCx}$ & No change in morphology \\
\hline & & PND 30, & & \\
\hline & & PND 60 & & \\
\hline Poly(I:C) & C57BL6 mice/ & PND 28, & & \\
\hline & & & & No change in morphology \\
\hline Iba1 & $5 \mathrm{mg} / \mathrm{kg}$ (i.v.) & PND & HPC & (cell soma area and primary \\
\hline (Giovanoli et al. & GD 17.5 & 140, & & or secondary branches) \\
\hline 2015) & & PND 600 & & \\
\hline
\end{tabular}




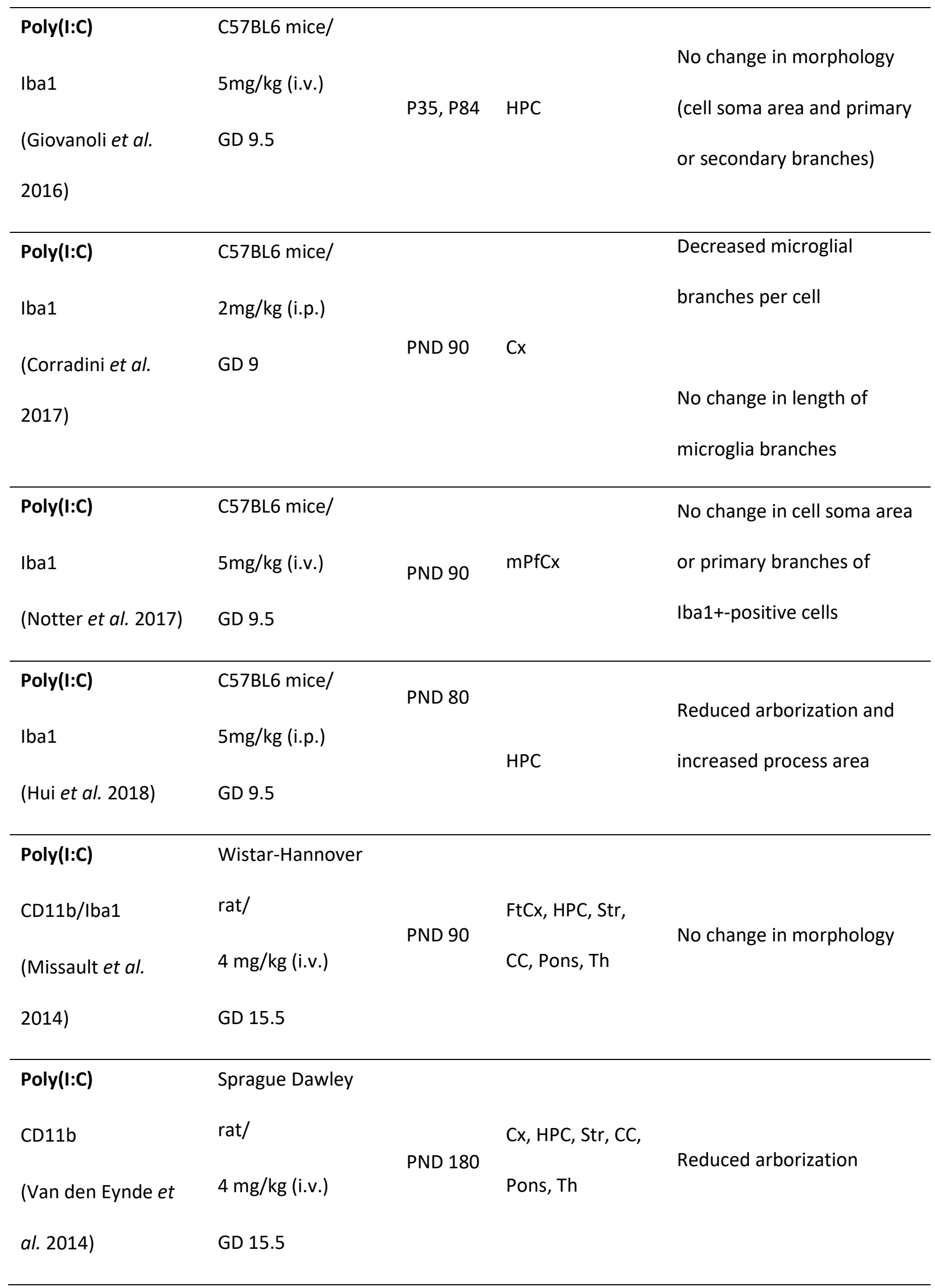




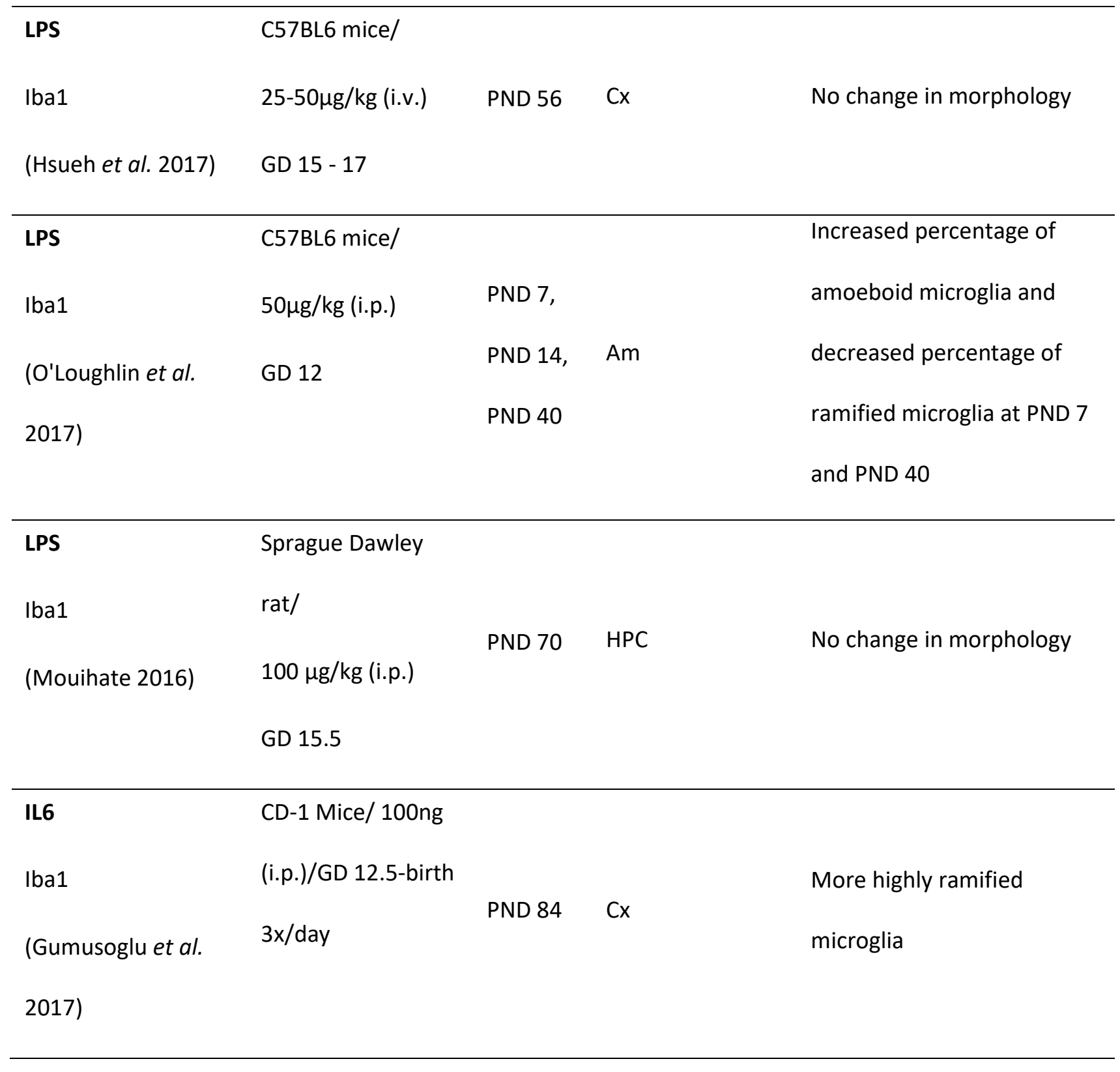

2 HPC: Hippocampus, Str: Striatum, Th: Thalamus, Cx: Cortex, $\mathrm{CgCx}$ : Cingulate Cortex, FtCx:

3 Frontotemporal Cortex, (m)PfCx: (medial) Prefrontal Cortex, CC: Corpus Callosum, Am: Amygdala

5 Expression of microglia activity dependent genes and proteins

6 The analysis of a single aspect in the continuum of microglia activation states is insufficient to capture

7 subtle changes in microglia reactivity, especially in the MIA model, where subtle changes in microglia 
activity have been detected using highly sensitive approaches including microglia-specific mRNA sequencing (Matcovitch-Natan et al. 2016). In order to capture subtle changes, one has to either apply microglia-specific protein or mRNA expression approaches or apply several complementary approaches. A widely used approach is to complement microglia density and morphological analyses with assessing the expression of microglial surface markers as well as cytokine and chemokine levels. Amongst the most widely used microglia markers are: CD68 (a lysosomal-associated membrane glycoprotein known to be upregulated in pro-inflammatory activity states of microglia), CD11b (subunit of the complement receptor 3 complex), MHCII, CX3CR1, CD200R (cell surface receptor of CD200, transmits inhibitory signals to microglia), and Iba1 as well as various different pro- (IL1 $\beta$, IL6, Tumor necrosis factor $\alpha(\mathrm{TNF} \alpha)$ ) and anti-inflammatory (IL-5, IL10) cytokine and chemokine levels.

Again, the findings in the expression pattern of microglia markers in MIA models are diverse (Table 3). In addition, not all studies have implemented all complementary methods described before which precludes the determination of a microglia phenotype to some extent. For example, one study reported a decreased immunoreactivity of Iba1 in the dendate gyrus of MIA offspring with no changes in cell density (Mattei et al. 2014). In contrast, another study found an increased immunoreactivity of Iba1 in the dendate gyrus upon MIA (Mattei et al. 2017). In the latter study, the interpretation of what Iba1 expression changes signify when presented isolated from other microglia analyses can be difficult. For example, an observed increase in Iba1 could signify increased expression of Iba1 by microglia, increased density of microglia, increased microglia soma size (indicative for pro-inflammatory state) or an increase in ramification. Hence, the combination of other measures such as Iba1 expression with microglia density (Mattei et al. 2014) can aid the interpretation of the obtained results. In this example, decreased Iba1 expression together with no changes in density could be an indicator for either decreased expression of Iba1, or altered morphology with less ramification and smaller soma size. Findings from other microglia marker expression studies have yielded similarly contradicting results. In summary, while 8 out of 13 studies found no change in the expression of CD68, CD11, or Iba1, 7 
1 studies did find changes in the expression (either increased or decreased) of these same markers and

2 others (MHCII, CD124, CX3CR1, etc.)(Table 3).

3 Two recent studies measured changes in the expression of the mitochondrial TSPO in the brains of MIA

4 offspring, similar to in vivo PET imaging studies in humans (Mattei et al. 2017, Notter et al. 2017). Both

5 studies find opposite results, with increased TSPO in one study and decreased TSPO in the other. The

6 reason for this discrepancy could be the difference in brain region (prefrontal cortex vs hippocampus)

7 or the difference in the MIA protocol (i.v. vs i.p. administration of Poly(I:C) at GD9 or GD17). As

8 discussed by the authors and recently reviewed in Notter et al. 2018, the use of TSPO to assess the

9 activation status of microglia is debatable as it is also expressed in other CNS cell types, including astrocytes and endothelial cells (Notter et al. 2017, Tronel et al. 2017, Notter et al. 2018). The increase

11 in TSPO could thus also signify processes other than inflammation, such as oxidative stress or cell

12 activity per se.

Table 3: Microglia surface marker expression in postnatal MIA offspring

\section{Microglia Surface Marker Expression}

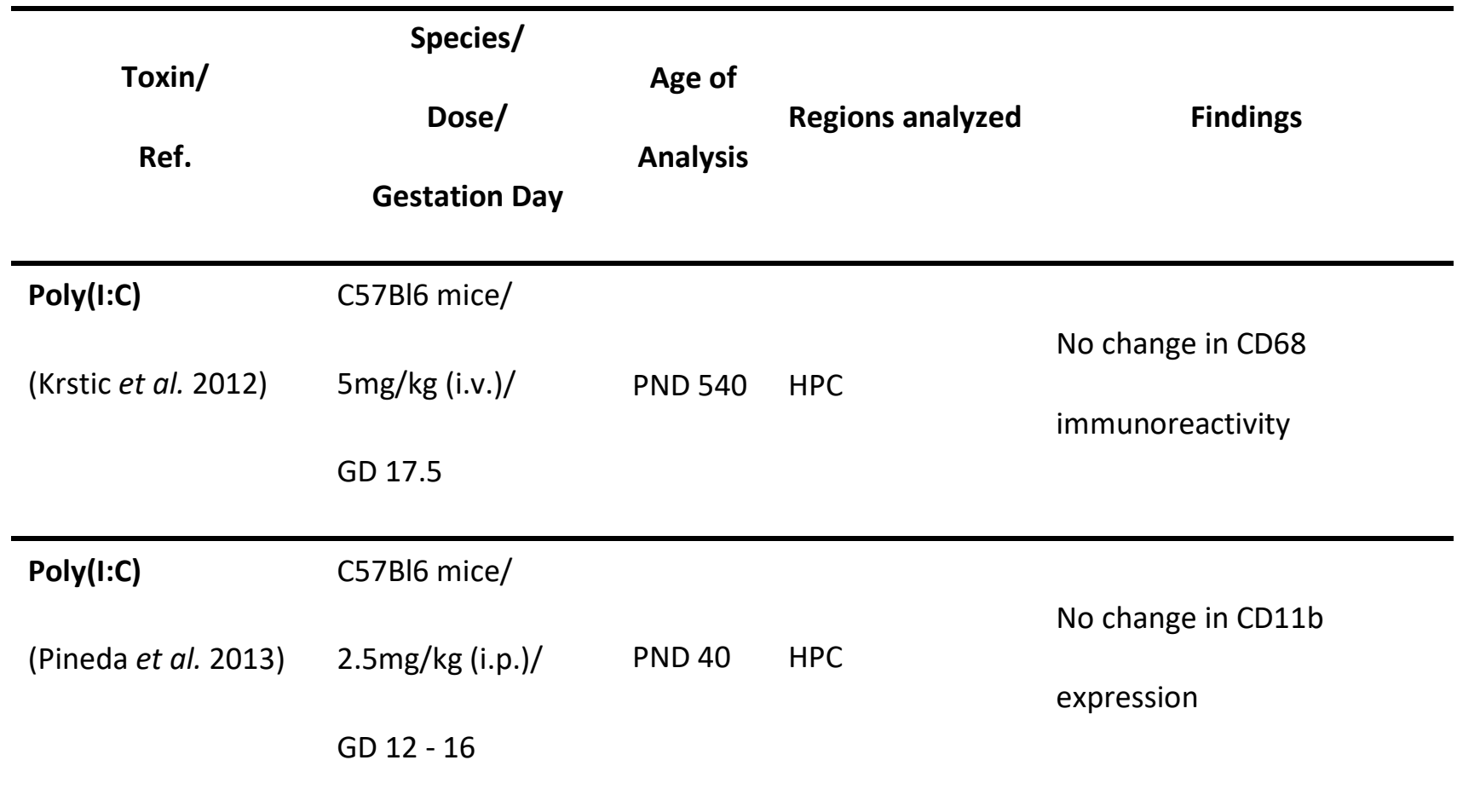




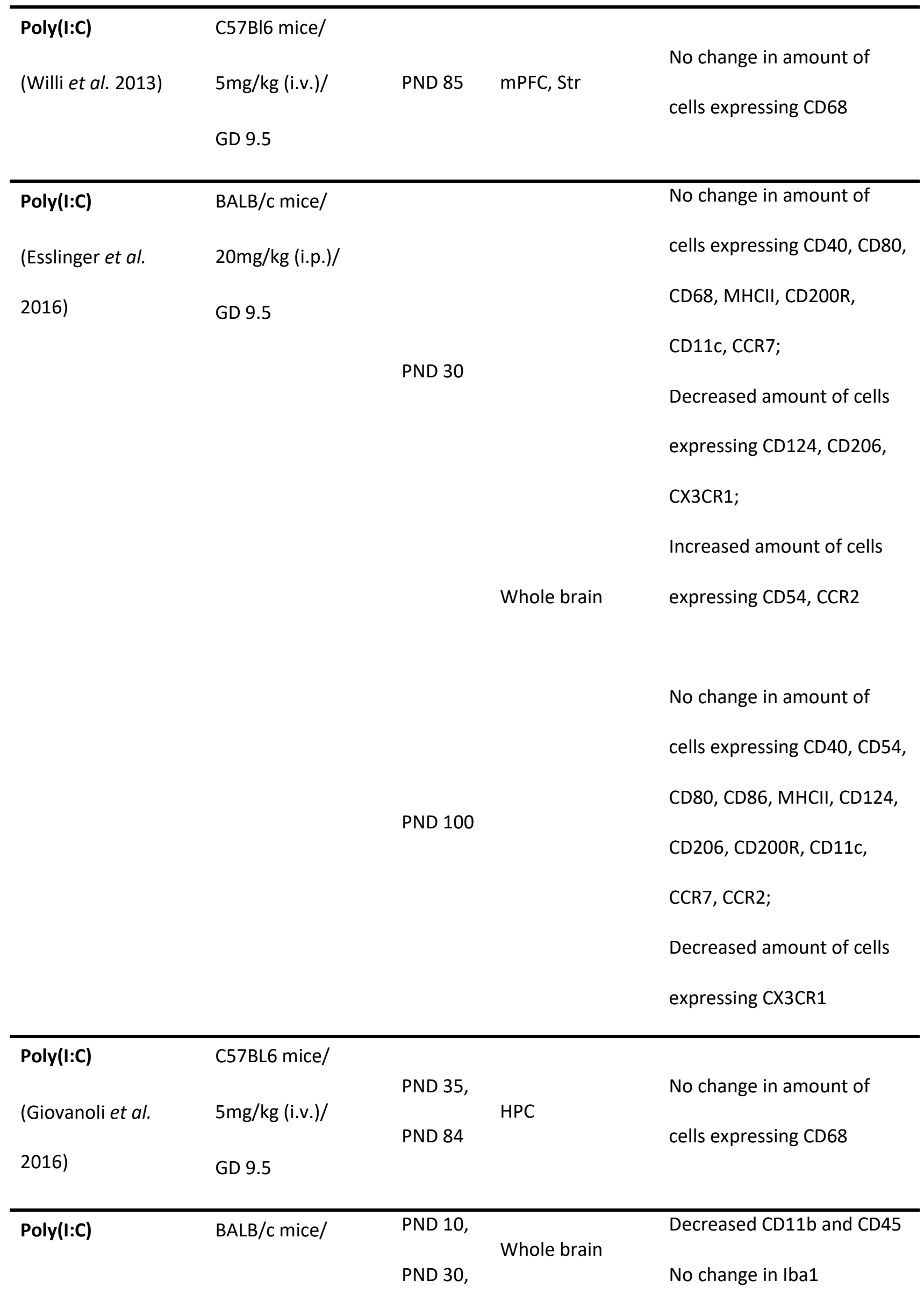




\begin{tabular}{|c|c|c|c|c|}
\hline \multirow[t]{2}{*}{ (Manitz et al. 2016) } & 20mg/kg (i.9.)/ & \multicolumn{3}{|l|}{ PND 100} \\
\hline & GD 9 & & & \\
\hline Poly(I:C) & C57BL6 mice/ & & & Increased Iba1 reactivity \\
\hline \multirow[t]{2}{*}{ (Mattei et al. 2017) } & $5 \mathrm{mg} / \mathrm{kg}$ (i.p.)/ & PND 120 & HPC & Increased TSPO binding \\
\hline & GD 15.5 & & & potential \\
\hline Poly(I:C) & C57BL6 mice/ & & & \\
\hline (Corradini et al. & $2 \mathrm{mg} / \mathrm{kg}$ (i.p.)/ & PND 90 & $C x$ & $\begin{array}{l}\text { No change in amount of } \\
\text { cells expressing CD } 11 b\end{array}$ \\
\hline 2017) & GD 9 & & & \\
\hline Poly(I:C) & C57BL6 mice/ & & & No change in CD68 optical \\
\hline \multirow[t]{2}{*}{ (Notter et al. 2017) } & $5 \mathrm{mg} / \mathrm{kg}($ i.v. $) /$ & PND 90 & mPFC, HPC & density \\
\hline & GD 9 & & & Decreased TSPO density \\
\hline Poly(I:C) & Wistar Rat/ & & & \\
\hline \multirow[t]{2}{*}{ (Mattei et al. 2014) } & $4 \mathrm{mg} / \mathrm{kg}$ (i.v.)/ & PND 128 & $\mathrm{HPC}, \mathrm{Cb}$ & Decreased Iba1 reactivity \\
\hline & GD 15.5 & & & \\
\hline \multirow[t]{2}{*}{ Poly(I:C) } & Sprague Dawley & & & \\
\hline & Rat/4mg/kg (i.v.)/ & PND 180 & $\mathrm{Cx}, \mathrm{HPC}, \mathrm{Str}, \mathrm{CC}$, & \\
\hline (Van den Eynde et & & & Pons, Th & reactivity \\
\hline al. 2014) & GD 15.5 & & & \\
\hline \multirow[t]{2}{*}{ LPS } & C57BL6 mice/ & & & \\
\hline & & & & No change in Iba1 optical \\
\hline \multirow[t]{2}{*}{ (Hsueh et al. 2017) } & 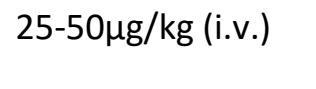 & PND 56 & $C x$ & density \\
\hline & GD $15-17$ & & & \\
\hline \multirow[t]{2}{*}{ LPS } & Wister rat/ & & & Increased MHCII \\
\hline & & P100 & Internal capsule & \\
\hline (Borrell et al. 2002) & $1 \mathrm{mg} / \mathrm{kg}$ (s.c.)/ & & & immunoreactivity \\
\hline
\end{tabular}


alternate days

1

HPC: Hippocampus, Str: Striatum, Th: Thalamus, Cx: Cortex, (m)PfCx: (medial) Prefrontal Cortex, CC:

\section{Corpus Callosum, Cb: Cerebellum}

Besides microglia surface markers, a number of studies have assessed the expression of pro- and antiinflammatory cytokines in MIA offspring (Table 4). Although not specific for microglia, several studies determined cytokine levels in brain homogenates in an attempt to give an idea about the inflammatory status of the brain. However, cytokines are not only produced by microglia, but also by other parenchymal cell types (such as astrocytes and neurons) and even by cells in the meningeal space. Moreover, many cytokines (such as IL1 $\beta$, IL6 and TNF $\alpha$ ) not only play a role in inflammation but also in modulation of synaptic transmission and plasticity (as reviewed in (Vezzani and Viviani 2015)). Cytokine levels can be measured either on the mRNA or on the protein level but differences in transcriptional and translational dynamics might create additional variation between studies. In total, 8 studies determined cytokine levels in brain homogenates and findings are divergent. Yet, this might also be explained by dynamic changes in several brain cytokines in a region- and age-specific manner of early postnatal to adult MIA offspring (PND $0-60$ ) (Garay et al. 2013). To our knowledge, only two studies measured cytokine levels (on the mRNA level) in isolated microglia from adult MIA offspring. Strikingly, although the protocols used to induce MIA and the age and brain region (hippocampus) analyzed are the same, Mattei et al. 2014 showed increased levels of IL1ß mRNA while Hadar et al. 2016 found no change in IL1ß mRNA levels (Mattei et al. 2014, Hadar et al. 2016).

\section{Table 4: Microglia cytokine expression in postnatal MIA offspring}

\section{Cytokine mRNA/protein Expression}




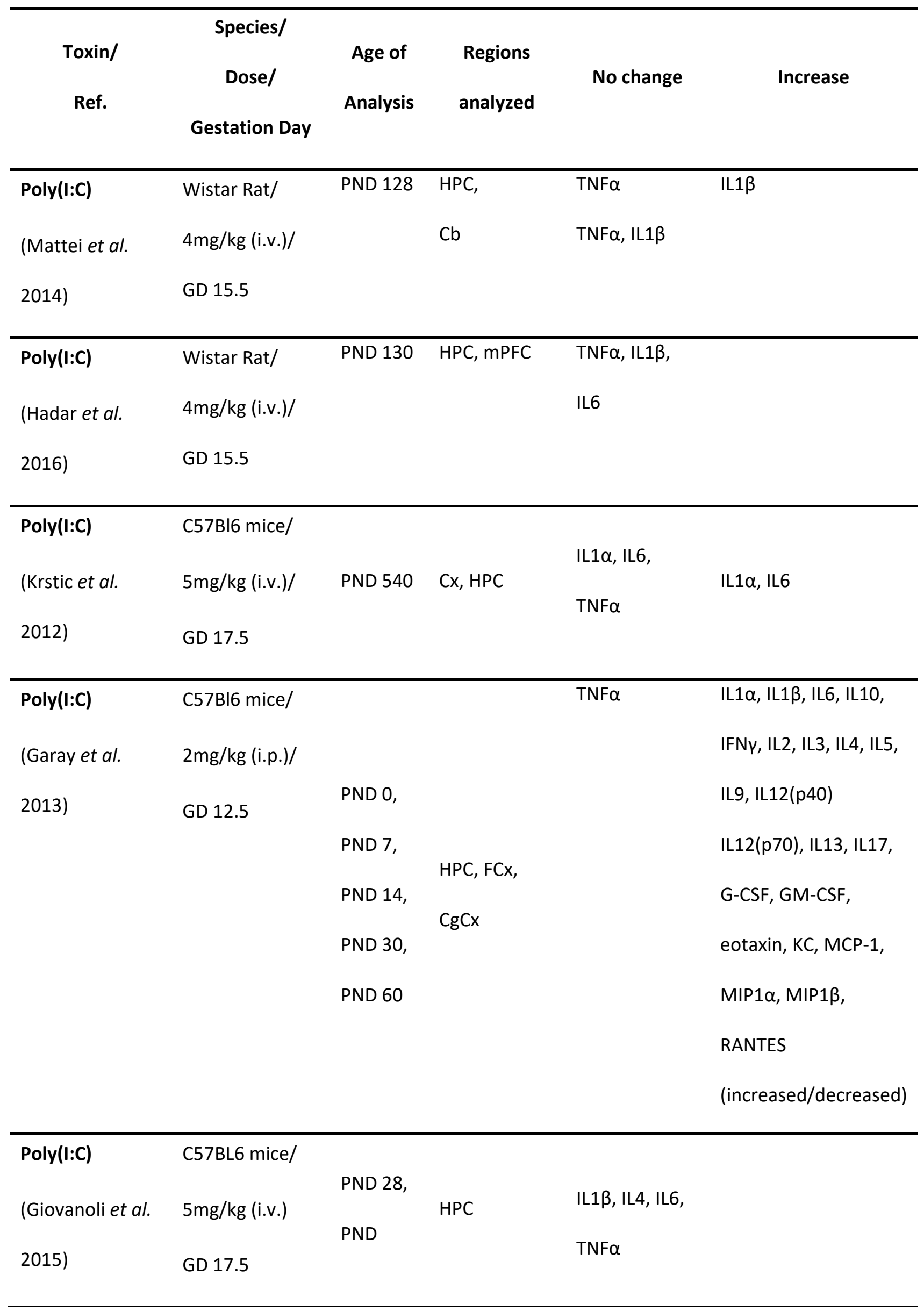


140,

PND 600

\begin{tabular}{|c|c|c|c|c|c|}
\hline Poly(I:C) & C57BL6 mice/ & PND 40, & & IL4, IL6, TNF $\alpha$, & \\
\hline (Giovanoli et al. & $5 \mathrm{mg} / \mathrm{kg}$ (i.v.) & & HPC & IL1及 & \\
\hline 2016) & GD 9 & PND 90 & & IL4, IL6, TNF $\alpha$ & IL1ß \\
\hline Poly(I:C) & C57BL6 mice/ & PND 90 & $\mathrm{mPfCx}$ & & \\
\hline (Notter et al. & $5 \mathrm{mg} / \mathrm{kg}$ (i.v.) & & & IL1 $\beta$, IL5, IL6 & IL10, TNF $\alpha$, IFN $\gamma$ \\
\hline 2017) & GD 9 & & & & \\
\hline
\end{tabular}

\begin{tabular}{|c|c|c|c|c|c|}
\hline Poly(I:C) & C57BL6 mice/ & PND 120 & $\mathrm{HPC}$ & & \\
\hline (Mattei et al. & $5 \mathrm{mg} / \mathrm{kg}$ (i.p.) & & & IL1 $\beta$, TNF $\alpha$ & IL6 \\
\hline 2017) & GD 15.5 & & & & \\
\hline
\end{tabular}

\begin{tabular}{|c|c|c|c|c|c|}
\hline Poly(I:C) & Sprague Dawley & PND 1 & Whole brain & NGF, BDNF & TNF $\alpha$ (decrease) \\
\hline (Gilmore et al. & $\mathrm{rat} / 10 \mathrm{mg} / \mathrm{kg}$ & PND 7 & $\mathrm{FCx}$ & NGF, BDNF, & \\
\hline 2005) & (i.p.) GD16 & & & TNF $\alpha$ & \\
\hline LPS & Sprague Dawley & PND 7 & $\mathrm{FCx}$ & & TNF $\alpha$ (decrease) \\
\hline (Gilmore et al. & rat/ $100 \mu \mathrm{g} / \mathrm{kg}$ & & & & \\
\hline 2005) & (i.p.) GD14 - 16 & & & & \\
\hline \multirow[t]{2}{*}{ LPS } & C57BL6 mice/ & PND 7 & & & IL1 $\beta$ \\
\hline & & & & IL1 $\beta$, IL6, IL10 & \\
\hline (O'Loughlin et & $50 \mu \mathrm{g} / \mathrm{kg}$ (i.p.) & PND 14 & $\mathrm{Am}$ & and/or TNF $\alpha$ & \\
\hline al. 2017) & GD 12 & PND 40 & & & \\
\hline
\end{tabular}


HPC: Hippocampus, Cx: Cortex, $\mathrm{CgCx}$ : Cingulate Cortex, FCx: Frontal Cortex, (m)PfCx: (medial) Prefrontal Cortex, Cb: Cerebellum, Am: Amygdala. The double line separates studies performed in isolated microglia (above) from those in brain homogenate (below).

By categorizing different studies according to their method for assessing microglia activation, we aimed to ease the comparison. However, contradictory results were found between different studies, which could, at least in part, be explained by the large variety in protocols used to induce MIA. However, even when comparing studies using comparable protocols for MIA induction, the results remain contradicting. For example, using an i.v. injection of Poly $(\mathrm{l}: \mathrm{C})(4 \mathrm{mg} / \mathrm{kg})$ at GD 15.5 in rats, 3 studies described increased microglial densities in several brain regions (Mattei et al. 2014, Van den Eynde et al. 2014, Hadar et al. 2016) while the same protocol did not induce changes in microglial density in 2 other studies (Missault et al. 2014, Paylor et al. 2016). Similarly, the same protocol induced no morphological changes at PND 90 in one study (Missault et al. 2014), while reduced arborization was observed in PND 180 offspring in another study (Van den Eynde et al. 2014). Despite applying the same MIA paradigm, these studies differ with regard to the age of the offspring when microglia were assessed, pointing towards a possible involvement of natural or pathological changes in cell density along the course of development

Taken together, when analyzing the effect of MIA on microglia in adult MIA-offspring, considerable controversy exists when only one parameter for microglia activation, i.e. density, morphology or expression of specific markers or cytokines, is assessed, even when comparing studies using the same or highly similar MIA induction protocols. In addition, the methods to detect 'microglia activation' described above are limited in their sensitivity and detect only obvious changes in microglia activation, and should therefore ideally be combined with more sensitive approaches, allowing the definition of distinct and very refined activity states. Although we conclude that morphology as a single readout is not sufficient to detect microglial activation, it is possible that subtle morphological changes are not detected with the standard microscopic analysis and in this aspect advanced and high resolution 
imaging such as electron microscopy might shed some more light. Moreover, using direct RNA sequencing, mRNA sequencing, gene and microRNA array, quantitative proteomic analysis and chromatin accessibility analysis, several groups have defined the unique molecular signature and 'sensome' of microglia and their regulatory dynamics during brain development (Hickman et al. 2013, Butovsky et al. 2014, Matcovitch-Natan et al. 2016, Hanamsagar et al. 2017, Thion et al. 2018)\}. Interestingly, using these techniques, Mattei et al. 2017 and Matcovitch-Natan et al. 2016 showed a MIA-induced deregulation of genes involved in microglial development, inflammation and phagocytosis accompanied by increased pro-inflammatory signaling and decreased phagocytic activity in microglia of MIA offspring (Matcovitch-Natan et al. 2016, Mattei et al. 2017), arguing in favor of combining several methods to more thoroughly define the effect of MIA on microglia.

\subsection{Readout at embryonic age}

Activation of adult microglia due to an embryonic inflammatory insult - as described in the section above - assumes some kind of activation memory. Much more strait forward is the hypothesis that embryonic microglia are activated at the time of maternal immune activation and thereby influence early neurodevelopment. The detection of increased mRNA levels of inflammatory cytokines in the embryonic brain within a few hours upon MIA (Meyer et al. 2006) strengthened the hypothesis that MIA could alter microglia activation states during prenatal development. Recently, the expression of sensome genes was shown already in the progenitor phase of microglia (yolk sac macrophages GD10.5) (Thion et al. 2018), lending even more support to the notion that microglia are already able to respond to (pathological) changes in the environment in early stages of development. In order to test this, the microglia activation phenotype has been studied during embryonic periods (Table 5). As for the findings in postnatal offspring, the results on embryonic microglia activation are divergent. For example, out of 6 studies that measured microglial density in embryonic MIA offspring, 3 studies measured no change while the other 3 studies detected increased microglial densities. Whilst other studies did not measure alterations in microglia density or morphology upon MIA, they measured 
altered gene and protein expression of inflammatory mediators in embryonic brain or microglia (Cunningham et al. 2013, Pratt et al. 2013, Pont-Lezica et al. 2014, O'Loughlin et al. 2017, Schaafsma et al. 2017). Similar to studies measuring cytokine levels in the brain of postnatal MIA-offspring, also several studies analyzing embryonic brains measured cytokines in brain homogenates rather than in isolated microglia. In this review, to exclude the possible contribution of maternally derived cytokines, we consider only studies measuring cytokines at least 48 hours after the immune challenge. As such, time-dependent changes in the levels of IL10, TNF $\alpha$ and IL1 $\beta$ were detected (O'Loughlin et al. 2017). To measure changes in microglia specifically, Pont-Lezica et al. 2014 used genome-wide gene expression profiling of isolated embryonic microglia and found a differential gene expression of 3906 genes upon maternal inflammation (Pont-Lezica et al. 2014). They found that several immune genes such as IL1 $\beta, \mathrm{C}-\mathrm{C}$ motif Chemokine ligand 4 (CCL4) and Nuclear factor (NF)-kB were upregulated while microglial genes involved in neuritogenesis such as Semaphorin (Sema)3c and Versican (Vcan) were downregulated (Pont-Lezica et al. 2014). Cunningham et al. 2013 performed immunohistochemical analyses on embryonic brain slices from rats exposed to LPS against markers indicative for different inflammatory activation states including inducible nitric oxide synthase (iNOS) and IL1 $\beta$ (proinflammatory state) or arginase-1 and IL-1RA (anti-inflammatory state) respectively. While the amount of microglia that expressed arginase-1 was not changed, the proportion of microglia expressing iNOS, IL1 $\beta$ or IL-1Ra was increased upon LPS administration (Cunningham et al. 2013). Similarly, repeated prenatal administration of LPS in mice increased mRNA levels for IL1 $\beta$, IL6 and TNF $\alpha$ in isolated microglia 3 hours after the last LPS injection (Schaafsma et al. 2017). Furthermore, Pratt et al. 2013 assessed both mRNA and protein levels of inflammatory mediators in isolated microglia from MIAexposed embryonic brains. They found increased levels of IL6 mRNA whereas MRNA levels for IL1 $\beta$, TNF $\alpha$ and IL10 were unchanged. On the protein level, they found increased expression of IL1 $\alpha$, IL4 and IL9 but not IL1 $\beta$, IL6, IL10 or TNF $\alpha$ (among others) (Pratt et al. 2013). The fact that mRNA levels for IL6 were increased while protein levels for this cytokine remained unchanged could be explained by differences in transcriptional and translational dynamics, as mentioned before. In contrast to these 
1 findings, no change in microglial protein levels of inflammatory markers including iNOS, IL1 $\beta$ or Mac2

2 was detected in the hippocampus and cortex of embryos at different developmental stages upon

3 Poly(I:C) exposure at GD 11.5 (Smolders et al. 2015). Also repeated immune stimulation (at GD 11.5

4 and GD 15.5) did not affect embryonic microglia at GD 17.5 (Smolders et al. 2015).

5 Table 5: Microglia alterations in embryonic MIA offspring

Microglia alterations in embryonic MIA offspring

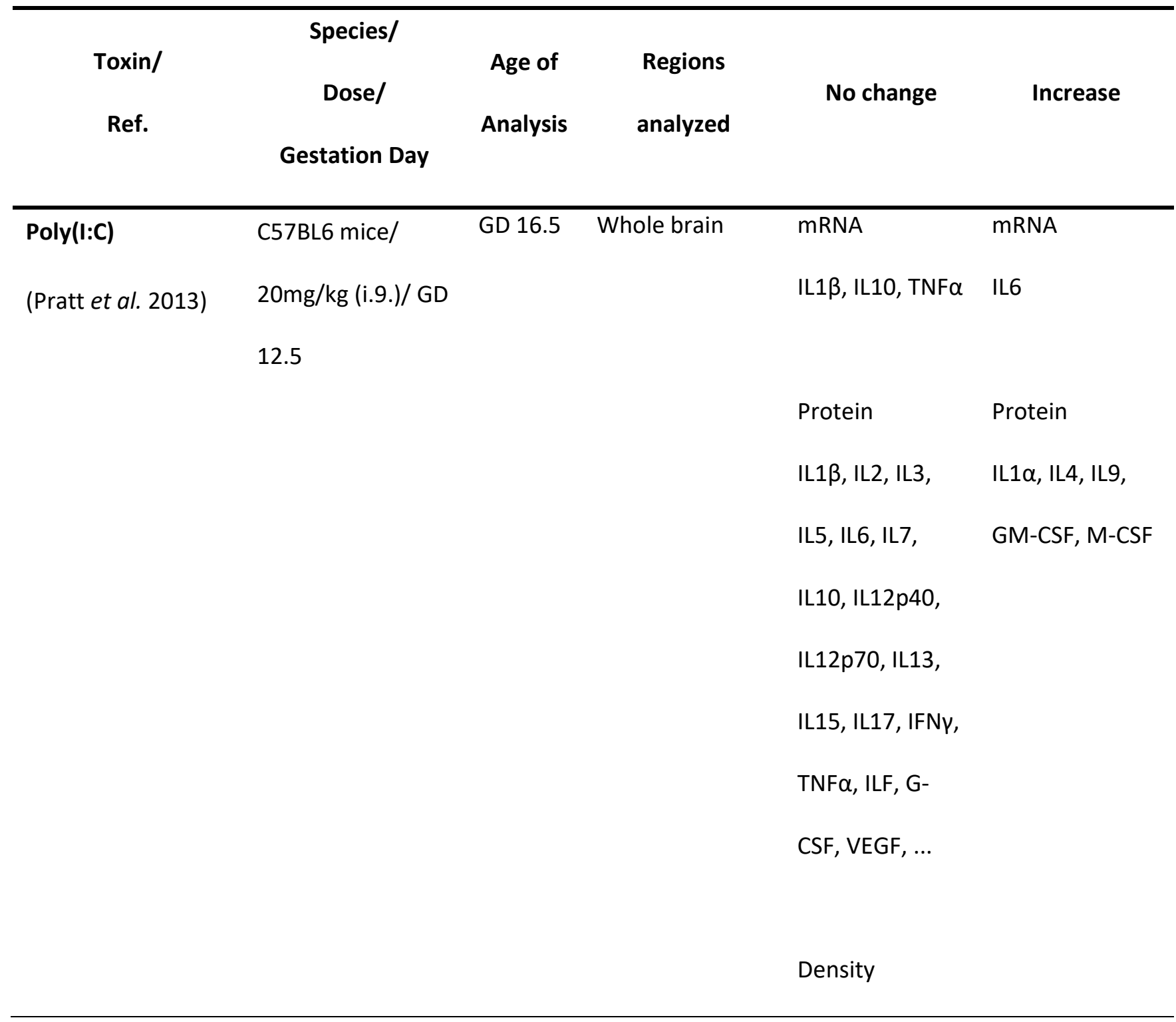




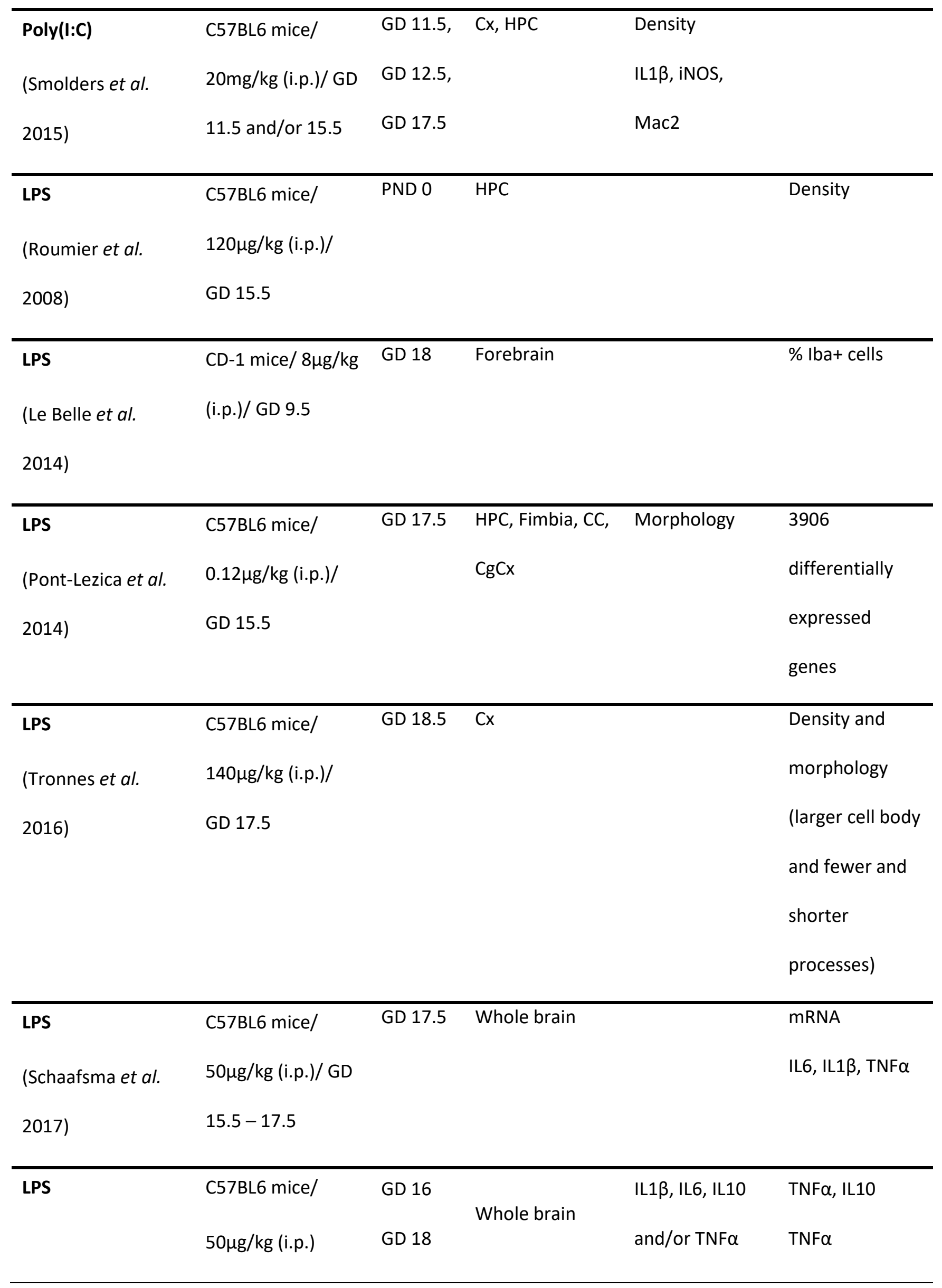




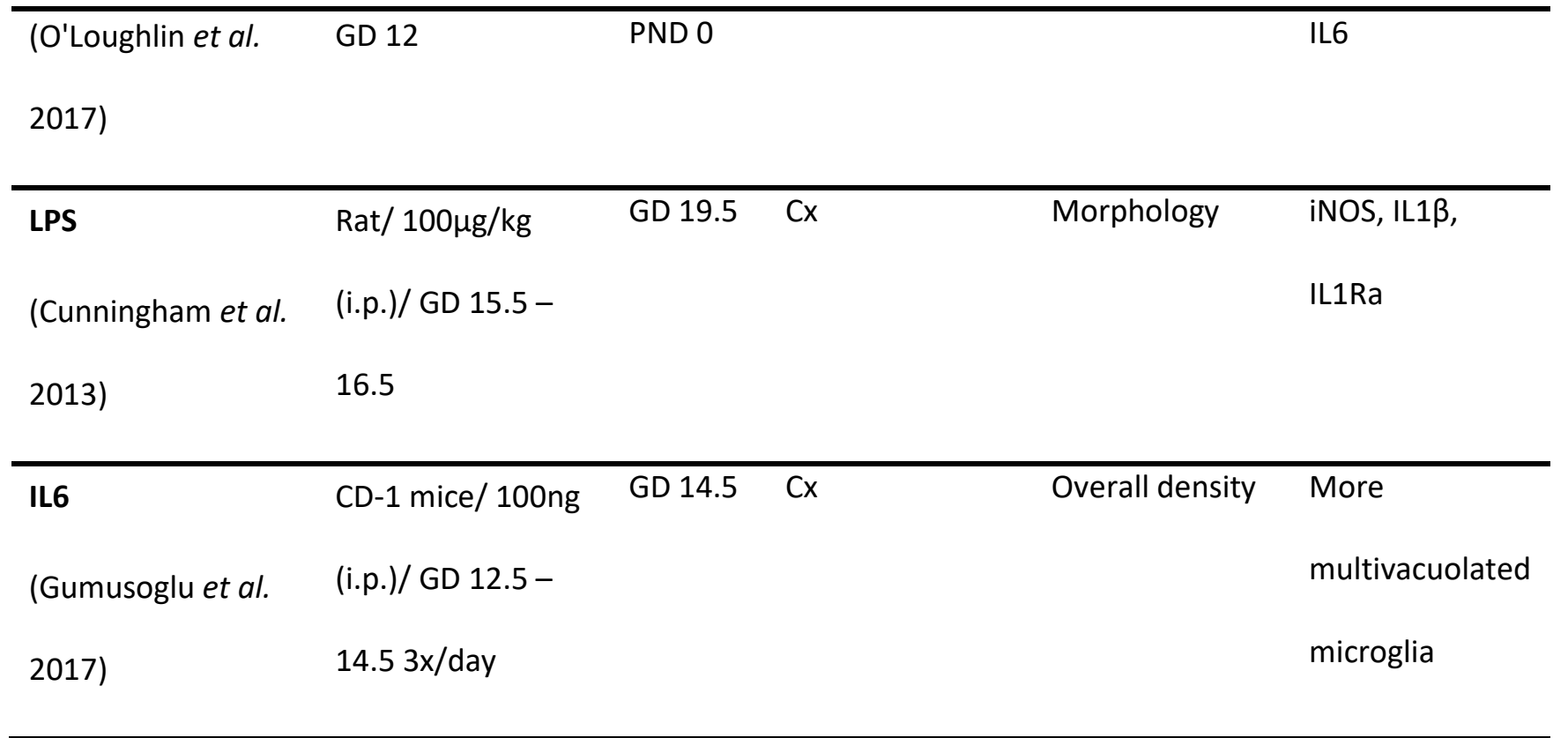

3 During rodent neurodevelopment, the brain environment substantially changes every day and 4 microglia are likely to adapt accordingly and even contribute to these changes (Cunningham et al. 5 2013, Matcovitch-Natan et al. 2016, Hanamsagar and Bilbo 2017). As a consequence, potential MIA6 induced alterations in microglia functions could be transient and its detection highly dependent on 7 when and where microglia phenotypes are assessed. This could in part explain some of the 8 controversial findings in microglia phenotypes described. For example Tronnes et al. 2016 found an 9 increased microglia density and altered microglia morphology in the somatosensory cortex 24 hours after MIA at GD 17.5 (Tronnes et al. 2016), whilst no evidence of altered microglia density was detected in the fetal cortex and hippocampus 24 hours after maternal injection of Poly(I:C) at GD 11.5 (Smolders et al. 2015). Again direct comparison between these two studies is problematic due to the difference in MIA paradigm and time of MIA induction. Nevertheless, others detected no changes in fetal microglia density or morphology 48-72 hours post-MIA induction (Cunningham et al. 2013, Pratt et al. 2013, Pont-Lezica et al. 2014, Smolders et al. 2015) whilst one study described an increase in the percentage of Iba1 positive cells 9 days post-LPS exposure (Le Belle et al. 2014). A similar controversy 
arises when microglial protein expression is assessed. For example, while Cunningham et al. 2013 found increased protein expression of iNOS and IL1 $\beta, 72$ hours after maternal LPS administration at GD 15.5 and GD 16.5, Smolders et al. 2015 found no change in the expression of these proteins 5, 24, 48 or 144 hours after maternal Poly(I:C) exposure at GD 11.5 or after a subsequent exposure at GD 15.5 (Cunningham et al. 2013, Smolders et al. 2015).

Taken together, clear conclusions cannot be drawn from the current data available on embryonic microglia phenotypes after MIA, firstly due to the omnipresent variety in the protocols applied (a similar phenomenon is seen in the studies assessing postnatal microglia phenotypes) and secondly due to the dynamic changes of microglia phenotypes during normal early development, which necessitates highly similar protocols of MIA induction and embryonic time points of analyses. However, as suggested by the study of Mattei et al. 2017, it can be concluded that embryonic microglia are able to sense and respond to MIA, causing long-term changes in their transcriptomic signature. This might indicate that the early embryonic insult deviates the phenotypical track of microglia, which in turn could also affect the development of other brain cells. Therefore, the characterization of MIA-induced changes in embryonic microglia at different developmental stages is crucial in order to understand how microglia could alter normal developmental trajectories that precipitate into the neuronal and behavioral deficits detected in MIA.

\section{Age, sex and brain region might affect microglial phenotype upon MIA}

In the previous section, we discussed the influence of the specific read-out to assess microglia activation (i.e. density, morphology, cytokine production and marker expression) on the precise outcome of the study. However, even when considering the same read-out, factors that are indirectly related to the analytical method will contribute to variability between studies. These factors include physiological constraints that alter the microglial phenotype such as age, sex and brain region. 


\subsection{Brain region-specific changes in microglia of MIA offspring}

In the normal brain, several microglial properties appear to be highly dependent on the specific brain region, including density, morphology, membrane properties and transcriptional profiles, possibly reflecting a different set of tasks in different brain regions (Schwarz et al. 2012, Yang et al. 2013, Grabert et al. 2016, De Biase et al. 2017). Moreover, a physiological function of microglia that received much attention in recent years, i.e. synaptic refinement, also appears to be region-dependent. In the hippocampus, elimination of synapses is dependent on CX3CR1 as mice lacking this receptor displayed increased hippocampal dendritic spine density accompanied by synaptic characteristics reminiscent of an immature connectivity and weak synaptic transmission (Paolicelli et al. 2011, Zhan et al. 2014). On the other hand, synaptic remodeling in the visual system was shown to be dependent on the complement system (in the lateral geniculate nucleus) and on P2Y12 (in the visual cortex) (Schafer et al. 2012, Sipe et al. 2016, Schecter et al. 2017). Hence, it should be of no surprise that environmental insults could affect microglia differently in different brain regions. In the context of MIA, several studies found changes in microglial density in one region while other regions were comparable to control mice upon Poly(I:C)-induced MIA (Juckel et al. 2011, Manitz et al. 2013, Mattei et al. 2014, Van den Eynde et al. 2014, Hadar et al. 2016, Hui et al. 2018).

\subsection{Age of MIA offspring}

Although schizophrenia and ASD share a subset of risk factors and symptoms (such as social interaction and communication deficits), they differ greatly in the age of disease onset. While ASD is usually diagnosed during childhood, schizophrenia typically emerges around adolescence. Similarly, the emergence of behavioral deficits appears to be age-dependent in the MIA model, which is effectively used to study the etiology of both ASD and schizophrenia. While deficits in prepulse inhibition, latent inhibition and amphetamine-induced activity are usually observed in adult MIA offspring (e.g. (Piontkewitz et al. 2011, Hadar et al. 2015, Fujita et al. 2016, Han et al. 2016)) (working) memory and 
social interaction deficits can readily be detected in pubescence (e.g. (Malkova et al. 2012, Fujita et al. 2016, Han et al. 2016)). Moreover, on the neuroanatomical level, decreases in hippocampal volume could be observed in pubescent MIA offspring while accelerated cortical thinning was only detectable in adulthood (Piontkewitz et al. 2011, Crum et al. 2017).

In the normal brain, microglial properties such as density and morphology change substantially over time (e.g. (Schwarz et al. 2012, Swinnen et al. 2013)). Also the transcriptional profile of microglia is highly dependent on age, possibly allowing microglia to perform specific functions at specifics stages (Grabert et al. 2016, Matcovitch-Natan et al. 2016, Thion et al. 2018). Finally, it was recently shown that the influence of the microbiome (as will be discussed later on in this review) on the microglial transcriptome is different according to age (with smaller influence in early development) (Thion et al. 2018)).

Consequently, effects of MIA on microglia might also be dependent on the age at which analysis was performed. Increased microglial densities were found in the hippocampus of Poly $(\mathrm{I}: \mathrm{C})$-induced MIA offspring between PND 2 and 30, which were no longer present in adulthood (Manitz et al. 2013, Li et al. 2014) while increased striatal microglia density was only observed in PND 100 offspring (Manitz et al. 2013). Furthermore, Esslinger et al. 2016 described changes in the expression of several microglial surface markers in whole-brain microglia from 30-day-old Poly(I:C)-MIA offspring, a phenotype that was lost when the offspring reached PND 100. A possible explanation for the loss of this phenotype could be the fact that microglia function changes during development - from progenitor pool maintenance in early brain development to synaptic remodeling during adolescence and adult brain homeostasis - and that the changes observed in microglia are related to the age-specific function of microglia at that specific point in time (Mosser et al. 2017).

\subsection{Sex of MIA offspring}

Neurodevelopmental disorders such as schizophrenia and ASD have a strong gender-bias, with a higher prevalence in males than in females (Abel et al. 2010, Mukherjee 2017). Although only a handful of 
studies directly compared the effects of MIA on behavior in both male and female offspring, most find a more severe behavioral deficit in males compared to females (Taylor et al. 2012, Naviaux et al. 2013, Xuan and Hampson 2014, Meehan et al. 2017, Hui et al. 2018). With regard to MIA studies investigating the effect on microglia, about one third of studies did not report on whether they analyzed males or females (e.g. (Roumier et al. 2008, Hsiao et al. 2012, Cunningham et al. 2013, Pratt et al. 2013)) and two thirds of studies used either males or a mix of males and females (not analyzed separately) (e.g. (Van den Eynde et al. 2014, Giovanoli et al. 2015, Hadar et al. 2016, Paylor et al. 2016)). Yet, several recent reports indicate substantial differences between microglial properties of both sexes, including microglia density and morphology, phagocytic activity and migration (Schwarz et al. 2012, Nelson et al. 2017, Weinhard et al. 2017, Yanguas-Casas et al. 2018). Also on the transcriptional level, differences have been described between male and female microglia. While the microglial transcriptome is similar during development, sexual dimorphism increases in adulthood (with female microglia in a more immune-activated state) (Hanamsagar et al. 2017, Thion et al. 2018). Moreover, the effects of postnatal immune challenge or of pre- and postnatal microbiome composition on the microglial transcriptome was dependent on sex (Hanamsagar et al. 2017, Thion et al. 2018). Hence, MIA might differentially affect male versus female microglia and thus result in divergent behavioral outcomes.

To our knowledge, only 3 studies directly compared the effects of MIA on microglia between male and female offspring. As such, Manitz et al. 2016 and Esslinger et al. 2016 showed sex-specific alterations in the expression of several proteins associated with microglial activation, including CD45, CD54, CD124 and CD206 ((Esslinger et al. 2016, Manitz et al. 2016)) in whole-brain lysates of Poly(I:C)-MIA offspring. Hui et al. 2018 recently also showed more pronounced effects of MIA (induced by Poly(I:C) on microglial distribution and arborization in the hippocampus of male offspring compared to females (Hui et al. 2018). Of interest, the studies described in this section all used Poly(I:C) to induce maternal inflammation when comparing the effects of MIA on microglia in different brain regions, between sexes or at different ages. Besides O'Loughlin et al. 2017, who identified microglial morphological 
changes at 3 different ages, no other studies that used LPS investigated the influence of the aforementioned factors.

Failure to report on the sex of the offspring examined is mostly prevalent in studies analyzing embryonic microglia, as sex determination by eye is not possible at this age. However, as done by Hsueh et al. 2017, genotyping of embryonic samples for the presence of the sex-specific SRY gene can readily distinguish between males and females at embryonic stages (Hsueh et al. 2017). Hence, the fact that microglia are measured at embryonic ages should not be a limitation to investigate gender differences.

\section{MIA-model associated factors can influence the study outcome}

As discussed in the previous section, different studies used different methodologies to determine microglia activation upon MIA, which could have led to the contradicting results on the effect of MIA on microglia. However, another notable difference between studies is the exact method used to induce MIA. Both Poly $(I: C)$ and LPS are administered at several doses and at different gestational ages, all of which are factors that could contribute to the variation in results that have been found by different studies. Moreover, lab specific factors such as housing conditions and food composition are likely to play an important role in the diversity between studies. In the following sections, we will discuss the possible influence of these factors on the outcome of studies determining microglia activation.

\section{$\underline{5.1 \text { Poly(l:C) vs LPS }}$}

The two most commonly used agents to induce MIA in rodents are LPS and Poly(I:C). MIA studies determining microglial alterations at postnatal or adult ages mostly used Poly $(\mathrm{I}: \mathrm{C})$ and only a few induced MIA with LPS. Regardless of which immune stimulant was used however, several studies suggest microglia "activation" in the adult MIA offspring while just as many studies find no evidence for changes in microglia. In contrast, studies assessing microglia "activation" at embryonic stages 
mostly used LPS to induce MIA with the exception of Pratt et al. 2013 and Smolders et al. 2015, who used Poly(I:C). Both Poly(I:C) and LPS elicit their effects via the toll like receptor (TLR) family, where Poly(I:C) signals via the TLR3 and LPS via TLR4. Unlike TLR4, which is located at the cellular membrane, TLR3 is localized to endosomal membranes. Although activation of both TRL3 and TLR4 seems to result in similar, but not equal, intracellular and cytokine responses, the precise set of immune mediators released by both immune activating agents has, to our knowledge, not been described. However, it seems likely that the different immune stimulants elicit the production and secretion of different subsets of cytokines (Reisinger et al. 2015, Bilbo et al. 2017). Indeed, specific cytokines have been attributed to play a critical role in both the Poly(I:C)-based and LPS-based MIA model. In 2007, Smith et al. showed that systemic injection of recombinant IL6 was able to evoke similar behavioral deficits as Poly $(\mathrm{I}: \mathrm{C})$ and that simultaneous injection of IL6 blocking antibody could prevent the MIA-induced behavioral changes (Smith et al. 2007). Complementary to this, Wu et al. 2017 showed that deletion of the IL6 receptor specifically in the placenta could prevent MIA-induced deficits in the offspring (Wu et al. 2017). Furthermore, it was shown that IL17a acts downstream of IL6 as a systemic injection of IL17a-antibody before Poly(I:C)-induced MIA was able to prevent altered ultrasonic vocalizations in MIA offspring (Choi et al. 2016). In addition to schizophrenia and ASD related symptoms, Pineda et al. 2013 also showed the importance of specific cytokines for epilepsy, a well-known co-morbidity in ASD patients. While the presence of only IL6 in maternal blood was sufficient to induce social impairment in the offspring, a combination of IL6 and IL1 $\beta$ was required to increase susceptibility to seizures (Pineda et al. 2013). In contrast to IL6, blocking IL1ß upon Poly(I:C) administration did not improve social deficits (Pineda et al. 2013). Although the previous studies described the importance of IL6 and IL17a in mediating the effects of Poly(I:C)-induced MIA on the offspring, IL1 was shown to be crucial for LPS-mediated effects (Girard et al. 2010) as co-administration of an IL1 receptor antagonist could alleviate LPS-induced placental damage, maintain microglia density and protect against motor dysfunction in the offspring. 
Beside the classical activation of TLR4, LPS was recently also shown to induce activation of transient receptor potential (TRP) channels in a TLR4-independent manner in airway epithelial cells and nociceptive sensory neurons (Meseguer et al. 2014, Alpizar et al. 2017). Activation of TRP channels in airway epithelial cells was speculated to be caused by mechanical perturbations induced by LPS in the plasma membrane and induced the production of bactericidal nitric oxide (Alpizar et al. 2017). Whether LPS also activates TRP channels in microglia is not known but, intriguingly, increased numbers of microglia expressing iNOS and IL1 $\beta$ were detected when acute embryonic brain slices were challenged with LPS whereas exposure to Poly(I:C) and recombinant IL6 failed to do the same (Smolders et al. 2015). These results raise the question whether LPS might have more potent effects both on microglia and other cells due to its additional activation of TRP channels.

In addition to specific cytokines induced by either Poly(I:C) or LPS, a different intensity of the immune response (either by administration of a higher dose or by repeated exposure) provoked by both immune stimulants could explain the differential effects of these 2 agents. Indeed, dose-response studies by Shi et al. 2003 and Meyer et al. 2005 showed that increasing doses of Poly(l:C) correlated with increased levels of cytokines in maternal blood and with increased severity of behavioral deficits in the offspring while increasing doses of LPS were able to induce abortion (Hsueh et al. 2017). As different batches of LPS and Poly(I:C) can differ in the intensity of immune response they induce, doseresponse studies are crucial and need to be performed. Moreover, the synthetic Poly(I:C) comes in different forms, low and high molecular weight or with added sodium salt, depending on the company, which could influence the precise dose. Hence, a clear statement on which Poly $(\mathrm{I}: \mathrm{C})$ was used and the exact dose should be included in every publication. Finally, a comparative study between LPS and Poly $(\mathrm{I}: \mathrm{C})$ addressing both classical immune activation and developmental functions of embryonic microglia would be of great value.

\subsection{Timing of MIA induction}


Besides the immune activating agent used to induce MIA, the embryonic age at which MIA is induced varies greatly between studies, ranging from early to mid (GD 9, GD 11, GD 12) and late gestation (GD 17). Intriguingly, studies comparing the behavioral deficits in offspring exposed to MIA during different gestational periods have shown that certain, but not all, behavioral phenotypes show a gestational dependency ((Meyer et al. 2006) and others). The precise mechanisms underlying this dependency remain to be fully identified (as discussed in detail in (Meyer et al. 2007)). The stage and ongoing processes of fetal development, as well as placental development have been suggested to play an important role. Besides this, microglial developmental stages could be perturbed differently depending on when MIA is induced, which could result in different perturbations of neurodevelopmental processes. Indeed recent findings of whole genome expression analyses of microglia isolated at different embryonic ages could identify distinct developmental stages of microglia (GD 10.5 - 14.5, GD 14.5 - PND 9 and PND 28) (Matcovitch-Natan et al. 2016). This could explain the findings described by Lai et al. 2013 where they detected an age-specific and activator-dependent activation profile of isolated, cultured microglia (Lai et al. 2013, Matcovitch-Natan et al. 2016). In line with this, Hanamsagar et al. 2017 identified a specific developmental maturation and immune reactivity profile of microglia in both mice and humans, which was shown to be gender- and agedependent (Hanamsagar et al. 2017). Hence, it seems plausible that microglia could be differentially perturbed depending on when MIA was induced which leads to differential changes in brain developmental processes and thus subtle changes in behavioral deficits later in life.

In addition to the immediate effects of MIA on microglia functions and early neurodevelopment, prenatal immune stimulation might prime embryonic microglia resulting in an exacerbation of their response to stimulants later in life (for example stress during childhood or puberty) and as such cause a deregulation in critical processes of brain maturation (Eggen et al. 2013, Knuesel et al. 2014, Slusarczyk et al. 2015, Giovanoli et al. 2016). Such priming effects were shown by Krstic et al. 2012 where an increased CD68 reactivity and changes in microglial morphology was observed in aged offspring that were exposed to two Poly $(\mathrm{I}: \mathrm{C})$ immune challenges, once during prenatal development 
1 (GD 17) and once during adulthood (Krstic et al. 2012). Giovanoli et al. 2013 and 2016 described

2 increased expression of CD68 and CD11b together with an enlargement of the microglial cell soma in

3 adolescent MIA offspring that was exposed to unpredictable stress during postnatal development

4 (Giovanoli et al. 2013, Giovanoli et al. 2016). Intriguingly, they further showed that minocycline treatment - a broad-spectrum tetracycline antibiotic that was shown to inhibit microglia activation during postnatal stress prevented the offspring to develop behavioral deficits (Giovanoli et al. 2016), pointing towards the importance of proper microglia function during postnatal periods for brain maturation, neuronal function and behavior.

\section{$\underline{5.3 \text { Housing, food, microbiome, strain and species effect on MIA outcome }}$}

As briefly touched upon in the introduction, several lab-specific factors that are not directly related to the protocol used to induce MIA in rodents might influence the response to MIA and contribute to the variation in study outcome we observe.

The two rodent species that are by far mostly used in the lab are mice and rats. However, it was recently shown by Lam et al. 2017 that the microglial response to inflammatory stimuli is different between these species (Lam et al. 2017). Even within a rodent species, different strains are being used to study the effect of MIA on microglia. A handful of studies reported a different vulnerability of inbred mouse strains, with different genetic background, to the effects of MIA (Schwartzer et al. 2013, Babri et al. 2014, Morais et al. 2018). Alterations in ultrasonic vocalizations, social interaction and repetitive behavior were more pronounced in BTBR mice compared to C57BL6 mice (Schwartzer et al. 2013). Similarly, maternal inflammation in NMRI or NIH Swiss mice induced more pronounced anxiety and depression-like behavior compared to C57BL6 mice (Babri et al. 2014, Morais et al. 2018).

Interestingly, Moraïs et al. 2017 showed that the increased vulnerability of NIH Swiss mice to effects of MIA on the offspring was accompanied by an increased gut permeability in adulthood, a defect that has previously been associated with microbiome dysregulation (Hsiao et al. 2013, Morais et al. 2018). Accumulating evidence suggests an important effect of microbiome composition, both from the 
mother and the offspring, on the development and homeostasis of microglia and the behavioral outcome of MIA. Adult germ-free mice or mice with limited microbiota complexity displayed global defects in microglia leading to an impaired innate immune response (Frick et al. 2013, MatcovitchNatan et al. 2016). Also in prenatal stages, absence of maternal gut microbiota resulted in dysregulated microglia transcriptomic profiles, altered microglial colonization of the embryonic brain (i.e. increased microglial density and excessive ramification) and increased chromatin accessibility (Thion et al. 2018).

The importance of microbiome composition in the context of MIA has also recently been addressed. Eradication of the vancomycin-susceptible segmented filamentous bacteria (SFB) prior to MIA induction prevented the emergence of behavioral deficits in the offspring (Kim et al. 2017). Additionally, maternal infection in C57BL6 mice obtained from Taconic Biosciences (which contain intestinal SFB) induced behavioral abnormalities in the offspring while the same protocol applied to C57BL6 mice from Jackson Laboratories (which are devoid of intestinal SFB) failed to elicit a behavioral phenotype (Kim et al. 2017). Finally, MIA was shown to induce a dysregulation of the offspring's microbiome which in turn was associated with behavioral symptom severity (Hsiao et al. 2013). Treatment of MIA offspring with microbiota partially prevented these gut alterations along with stereotypical and anxiety-like behavior and deficits in sensorimotor gating (Hsiao et al. 2013).

Another variable between labs is the composition of the food provided to rodents. Nevertheless, the composition of different food pellets can differ dramatically and influence the outcome of MIA studies. One important aspect of rodent food pellets is the amount of anti-inflammatory dietary omega 3 polyunsaturated fatty acids (PUFA) relative to the amount of pro-inflammatory omega 6 PUFAs. The importance of dietary omega 3 PUFA for brain development has been documented in rodents: deficiency during development in rodents leads to deficits in cognition, vision and a wide array of behaviors (Fedorova et al. 2009, Brenna 2011, Weiser et al. 2015) while feeding mice with diets high in omega 6 PUFA during gestation produces autistic-like features in offspring (Jones et al. 2013). In the context of MIA, Li et al. 2015 and Weiser et al. 2016 showed that omega 3 PUFA-enriched or 
docosahexaenoic acid-supplemented diet could prevent the onset of behavioral deficits in MIA offspring (Li et al. 2015, Weiser et al. 2016). Given the anti-inflammatory properties of omega 3 PUFAs, it seems plausible that the preventive effects could partially be explained by reduced microglial activation.

Finally, housing conditions can influence the effect of MIA on offspring's microglial and behavioral phenotype. In the study of Buschert et al. 2016, MIA offspring was either housed in standard conditions or in an environmentally enriched cage. Although no typical MIA-induced behavioral changes were observed, prenatal MIA reduced overall exploration (in the sociability test) in the environmentally enriched group, while it failed to do so in the standard housed animals as exploration. Similarly, a decrease in cortical microglial density upon MIA (which is in sharp contrast to the studies discussed in this review describing either unchanged or increased density) could only be observed in mice housed in an enriched cage, while both $\mathrm{NaCl}$ and Poly $(\mathrm{I}: \mathrm{C})$-exposed mice housed in a standard cage displayed decreased microglial density compared to the environmental enrichment group. The increase in microglial density, which is often interpreted as a sign of inflammation, upon housing in an enriched environment seems to contradict the findings of an anti-inflammatory effect of an enriched environment in disease contexts (including Alzheimer and Depression) (Williamson et al. 2012, Chabry et al. 2015, Xu et al. 2016). However, as shown by Ziv et al. 2006 and speculated by Buschert et al. 2016, these microglia might be in a neuroprotective rather than an inflammatory state (Ziv et al. 2006, Buschert et al. 2016). Taken together, these findings suggest that standard housing conditions can mask behavioral deficits and possibly microglial alterations induced by MIA.

In conclusion, we want to stress that authors should mention as much methodological details as possible in the materials and methods section concerning strain, housing, food etc., as they all have been shown to be relevant for the exact outcome of MIA studies and as a consequence possibly for the microglial response to MIA. 


\section{6. Alterations in microglia beyond dysregulated immune functions}

2 Over the last decade there has been a substantial shift in our understanding of the functional role of

3 microglia with regard to their non-immunological functions. Microglia are the first glial cells present in

4 the developing brain and have been attributed to play a role in neurogenesis, angiogenesis, 5 myelination, synaptogenesis and synaptic pruning (Bilimoria and Stevens 2015, Reemst et al. 2016). Although these functions are considered "non-immune" functions, some of them still rely on immune components, e.g. synaptic pruning has been shown to depend on the complement system (Hong et al. 2016). The fact that microglia originate from primitive macrophages and exert classical immune functions in response to insults of the CNS in the adult brain has led to the assumption that embryonic microglia exert similar roles in the developing brain. However, although equipped with immune related machinery, their functional contribution during development was found to exceed that of classical immune functions.

In support of this, microglia depletion during the first two weeks after birth was shown to result in long-lasting behavioral deficits (VanRyzin et al. 2016). Furthermore, transcriptomic studies showed that microglia are able to adapt according to regional cues, which translates in a regional and agedependent density, morphology, surface molecule expression and transcriptome profile of microglia (Hart et al. 2012, Schwarz et al. 2012, Butovsky et al. 2014, Grabert et al. 2016, De Biase et al. 2017, Mosser et al. 2017). In fact, microglia might adjust their functional phenotype as a response to developmentally changing factors in the neuro-environment to be able to perform their specific developmental task (Mosser et al. 2017). Hence, although studies investigating classical activation of microglia upon MIA are highly valuable, it might be more appropriate to look at gene profiles involved in the neurodevelopmental function of microglia, combined with assessing the related microglial functions during brain development. Indeed, recent studies point to MIA-induced changes in the microglial epigenome (Kaminska et al. 2016, Nardone and Elliott 2016) and transcriptome concerning genes involved in the developmental functions of microglia, such as genes involved in synaptogenesis 
and neuronal network formation (Pont-Lezica et al. 2014, Squarzoni et al. 2014, Matcovitch-Natan et al. 2016, Mattei et al. 2017). Matcovitch-Natan et al. described that microglia from mice subjected to MIA and analyzed from GD 14 to early postnatal weeks were transcriptionally shifted toward a more advanced developmental stage (Matcovitch-Natan et al. 2016). This implies that MIA renders microglia more "mature", which is in accordance with a recent microglial transcriptomics study suggesting an accelerated development of these cells in autism patients (Hanamsagar et al. 2017).

In support of altered neurodevelopmental microglial functions upon MIA, several reports describe increased phagocytosis of neuronal progenitor cells (Cunningham et al. 2013), a decrease in the outgrowth of dopaminergic axons (Squarzoni et al. 2014) and defasciculation of dorsal callosal axons (Pont-Lezica et al. 2014) in the embryonic brain of MIA offspring. In the latter case, mice lacking DNAXActivation Protein 12 (DAP12), a transmembrane protein that together with Triggering receptor expressed on myeloid cells 2 (TREM2) forms an immunoreceptor signaling complex and is associated with Nasu-Hakola disease, displayed similar deficits as observed after MIA. Moreover, MIA led to transcriptional alterations in microglia with regard to genes involved in growth of neurites, underscoring a disturbed developmental function of microglia after MIA (Pont-Lezica et al. 2014). Until now, conclusive experimental evidence for a direct, causative role of microglia in neurodevelopmental disorders after MIA is lacking. However, a recent study by Mattei et al. 2017 showed that MIA-induced changes in the microglial transcriptome and phagocytic function were accompanied by behavioral abnormalities (Mattei et al. 2017). Most interestingly, treatment of adult MIA offspring with minocycline rescued the transcriptional, functional and behavioral deficits. This study is among the first to point in the direction of dysfunctioning microglia as the culprits in inducing neurodevelopmental disorders upon MIA. Furthermore, disturbance of microglia-related proteins in humans such as Colony stimulating factor 1 receptor (CSF1r), TREM2, Methyl CpG binding protein 2 (MecP2), CD33, Interferon regulatory factor 8 (Irf8), P2x7r, DAP12 and Complement component 4 (C4) are associated with an increased likelihood to develop a plethora of neurological diseases, including schizophrenia and possibly autism ((Sekar et al. 2016) and reviewed in (Frost and Schafer 2016)). These 
data suggest that disturbance of specific functions of microglia during neurodevelopment correlate with disease outcome.

Taken together, microglia display specific and constantly changing transcriptional signatures throughout development and these profiles appear to be altered upon MIA. Although conclusive evidence to pinpoint microglial alterations as the cause for neurodevelopmental disorders is missing, accumulating evidence strongly suggests at least a partially shared causative role for microglia in MIAinduced cognitive and behavioral deficits.

\section{Conclusion}

In the search for evidence of altered microglia function as mediators for the neurological deficits caused by MIA, a lack of consistency is encountered. Several factors might explain these contradictory results and thereby add complexity to the comparison of different MIA studies with regard to microglial alterations. In this review, we addressed this issue of variability in the field and here we suggest guidelines that might result in convergence on findings with regard to the effects of MIA on microglia. On one hand, the exact protocol used to induce MIA in rodents differs substantially between studies, using different immune stimulants (mostly LPS or Poly(I:C)) at different doses and administration routes and administered at various gestational time points. These factors will result in a different environment to which the embryo is exposed and might differentially affect microglia. First, we want to urge researchers to fully state all experimental details (such as animal strain, housing conditions, food, sex, etc.) when reporting on the outcome of MIA studies. Secondly, we suggest to perform doseresponse studies of the immune stimulant used before the start of the study, in order to better control the inflammatory environment that is created. Although behavioral testing would be the most ideal read-out for these dose-response studies, measurements of cytokines such as IL6 or IL17a in maternal serum might provide the minimal amount of information on the extent of maternal inflammation. 
Finally, care should be taken to use the least invasive and stressful administration route, as stress could be an additional confounding factor for the outcome of the experiments.

On the other hand, substantial variability could be caused by the precise readout to assess "microglia activation" with respect to their immunological function, such as microglia density, morphology or immune marker expression, but also by the time point and brain region where microglia were analyzed. While many studies used only one of these read-outs to analyze the effect of MIA on microglia, we suggest to use a combination of several complementary methods to better define the precise changes observed in microglia. Moreover, although these relatively crude measures can aid our understanding of the role of microglia in MIA models to some extent, they might not be suitable to detect less obvious changes in microglia function related to developmental processes. Therefore, we believe future research on immune disturbance of neurodevelopment will benefit from a holistic approach in which developmental functions of microglia are studied using a combinatorial approach i.e. using readouts at transcriptional, protein and functional level - to study both intrinsic microglial characteristics and the neuron-microglia interaction.

\section{Acknowledgements}

The authors thank the Research Foundation - Flanders (FWO) for funding the MIA-related research (project nr G.0A05.13N).

\section{References}

Abel, K. M., et al. (2010). "Sex differences in schizophrenia." Int Rev Psychiatry 22(5): 417-428.

Alpizar, Y. A., et al. (2017). "TRPV4 activation triggers protective responses to bacterial lipopolysaccharides in airway epithelial cells." Nat Commun 8(1): 1059.

Antonson, A. M., et al. (2017). "Maternal viral infection during pregnancy elicits anti-social behavior in neonatal piglet offspring independent of postnatal microglial cell activation." Brain Behav Immun 59: 300-312. 
Arad, M., et al. (2017). "Immune activation in lactating dams alters sucklings' brain cytokines and produces non-overlapping behavioral deficits in adult female and male offspring: A novel neurodevelopmental model of sex-specific psychopathology." Brain Behav Immun 63: 35-49.

Arnold, T. and C. Betsholtz (2013). "Correction: The importance of microglia in the development of the vasculature in the central nervous system." Vasc Cell 5(1): 12.

Arsenault, D., et al. (2014). "The different effects of LPS and poly I:C prenatal immune challenges on the behavior, development and inflammatory responses in pregnant mice and their offspring." Brain Behav Immun 38: 77-90.

Babri, S., et al. (2014). "Strain-dependent effects of prenatal maternal immune activation on anxietyand depression-like behaviors in offspring." Brain Behav Immun 37: 164-176.

Bennett, M. L., et al. (2016). "New tools for studying microglia in the mouse and human CNS." Proc Natl Acad Sci U S A 113(12): E1738-1746.

Bilbo, S. D., et al. (2017). "Beyond infection - Maternal immune activation by environmental factors, microglial development, and relevance for autism spectrum disorders." Exp Neurol.

Bilimoria, P. M. and B. Stevens (2015). "Microglia function during brain development: New insights from animal models." Brain Res 1617: 7-17.

Boksa, P. (2010). "Effects of prenatal infection on brain development and behavior: a review of findings from animal models." Brain Behav Immun 24(6): 881-897.

Borrell, J., et al. (2002). "Prenatal immune challenge disrupts sensorimotor gating in adult rats. Implications for the etiopathogenesis of schizophrenia." Neuropsychopharmacology 26(2): 204-215.

Brenna, J. T. (2011). "Animal studies of the functional consequences of suboptimal polyunsaturated fatty acid status during pregnancy, lactation and early post-natal life." Matern Child Nutr $\mathbf{7}$ Suppl 2: 59-79.

Brown, A. S., et al. (2001). "A.E. Bennett Research Award. Prenatal rubella, premorbid abnormalities, and adult schizophrenia." Biol Psychiatry 49(6): 473-486.

Buka, S. L., et al. (2001). "Maternal infections and subsequent psychosis among offspring." Arch Gen Psychiatry 58(11): 1032-1037.

Buschert, J., et al. (2016). "Prenatal immune activation in mice blocks the effects of environmental enrichment on exploratory behavior and microglia density." Prog Neuropsychopharmacol Biol Psychiatry 67: 10-20.

Butovsky, O., et al. (2014). "Identification of a unique TGF-beta-dependent molecular and functional signature in microglia." Nat Neurosci 17(1): 131-143.

Buttgereit, A., et al. (2016). "Sall1 is a transcriptional regulator defining microglia identity and function." Nat Immunol 17(12): 1397-1406.

Careaga, M., et al. (2017). "Maternal Immune Activation and Autism Spectrum Disorder: From Rodents to Nonhuman and Human Primates." Biol Psychiatry 81(5): 391-401. 
Chabry, J., et al. (2015). "Enriched environment decreases microglia and brain macrophages inflammatory phenotypes through adiponectin-dependent mechanisms: Relevance to depressive-like behavior." Brain Behav Immun 50: 275-287.

Choi, G. B., et al. (2016). "The maternal interleukin-17a pathway in mice promotes autism-like phenotypes in offspring." Science 351(6276): 933-939.

Corradini, I., et al. (2017). "Maternal Immune Activation Delays Excitatory-to-Inhibitory GammaAminobutyric Acid Switch in Offspring." Biol Psychiatry.

Crotti, A. and R. M. Ransohoff (2016). "Microglial Physiology and Pathophysiology: Insights from Genome-wide Transcriptional Profiling." Immunity 44(3): 505-515.

Crum, W. R., et al. (2017). "Evolution of structural abnormalities in the rat brain following in utero exposure to maternal immune activation: A longitudinal in vivo MRI study." Brain Behav Immun 63: 50-59.

Cunningham, C. L., et al. (2013). "Microglia regulate the number of neural precursor cells in the developing cerebral cortex." J Neurosci 33(10): 4216-4233.

De Biase, L. M., et al. (2017). "Local Cues Establish and Maintain Region-Specific Phenotypes of Basal Ganglia Microglia." Neuron.

De Biase, L. M., et al. (2017). "Local Cues Establish and Maintain Region-Specific Phenotypes of Basal Ganglia Microglia." Neuron 95(2): 341-356 e346.

Eggen, B. J., et al. (2013). "Microglial phenotype and adaptation." J Neuroimmune Pharmacol 8(4): 807-823.

Esslinger, M., et al. (2016). "Schizophrenia associated sensory gating deficits develop after adolescent microglia activation." Brain Behav Immun 58: 99-106.

Fatemi, S. H., et al. (1998). "Differential expression of synaptosome-associated protein 25 kDa [SNAP25] in hippocampi of neonatal mice following exposure to human influenza virus in utero." Brain Res 800(1): 1-9.

Fedorova, I., et al. (2009). "Deficit in prepulse inhibition in mice caused by dietary n-3 fatty acid deficiency." Behav Neurosci 123(6): 1218-1225.

Frick, L. R., et al. (2013). "Microglial dysregulation in psychiatric disease." Clin Dev Immunol 2013: 608654.

Frost, J. L. and D. P. Schafer (2016). "Microglia: Architects of the Developing Nervous System." Trends Cell Biol 26(8): 587-597.

Fujita, Y., et al. (2016). "Supplementation with D-serine prevents the onset of cognitive deficits in adult offspring after maternal immune activation." Sci Rep 6: 37261.

Garay, P. A., et al. (2013). "Maternal immune activation causes age- and region-specific changes in brain cytokines in offspring throughout development." Brain Behav Immun 31: 54-68. 
Gilmore, J. H., et al. (2005). "Maternal poly I:C exposure during pregnancy regulates TNF alpha, BDNF, and NGF expression in neonatal brain and the maternal-fetal unit of the rat." J Neuroimmunol 159(12): 106-112.

Ginhoux, F., et al. (2010). "Fate mapping analysis reveals that adult microglia derive from primitive macrophages." Science 330(6005): 841-845.

Giovanoli, S., et al. (2016). "Preventive effects of minocycline in a neurodevelopmental two-hit model with relevance to schizophrenia." Transl Psychiatry 6: e772.

Giovanoli, S., et al. (2013). "Stress in puberty unmasks latent neuropathological consequences of prenatal immune activation in mice." Science 339(6123): 1095-1099.

Giovanoli, S., et al. (2015). "Late prenatal immune activation causes hippocampal deficits in the absence of persistent inflammation across aging." J Neuroinflammation 12: 221.

Giovanoli, S., et al. (2016). "Prenatal immune activation causes hippocampal synaptic deficits in the absence of overt microglia anomalies." Brain Behav Immun 55: 25-38.

Girard, S., et al. (2010). "IL-1 receptor antagonist protects against placental and neurodevelopmental defects induced by maternal inflammation." J Immunol 184(7): 3997-4005.

Grabert, K., et al. (2016). "Microglial brain region-dependent diversity and selective regional sensitivities to aging." Nat Neurosci 19(3): 504-516.

Gumusoglu, S. B., et al. (2017). "The role of IL-6 in neurodevelopment after prenatal stress." Brain Behav Immun.

Hadar, R., et al. (2016). "Deep brain stimulation during early adolescence prevents microglial alterations in a model of maternal immune activation." Brain Behav Immun.

Hadar, R., et al. (2015). "Using a maternal immune stimulation model of schizophrenia to study behavioral and neurobiological alterations over the developmental course." Schizophr Res 166(1-3): 238-247.

Han, M., et al. (2016). "Intake of 7,8-Dihydroxyflavone During Juvenile and Adolescent Stages Prevents Onset of Psychosis in Adult Offspring After Maternal Immune Activation." Sci Rep 6: 36087.

Hanamsagar, R., et al. (2017). "Generation of a microglial developmental index in mice and in humans reveals a sex difference in maturation and immune reactivity." Glia 65(9): 1504-1520.

Hanamsagar, R., et al. (2017). "Generation of a microglial developmental index in mice and in humans reveals a sex difference in maturation and immune reactivity." Glia.

Hanamsagar, R. and S. D. Bilbo (2017). "Environment matters: microglia function and dysfunction in a changing world." Curr Opin Neurobiol 47: 146-155.

Hanisch, U. K. and H. Kettenmann (2007). "Microglia: active sensor and versatile effector cells in the normal and pathologic brain." Nat Neurosci 10(11): 1387-1394.

Harry, G. J. and A. D. Kraft (2012). "Microglia in the developing brain: A potential target with lifetime effects." Neurotoxicology 33(2): 191-206. 
Hart, A. D., et al. (2012). "Age related changes in microglial phenotype vary between CNS regions: grey versus white matter differences." Brain Behav Immun 26(5): 754-765.

Hickman, S. E., et al. (2013). "The microglial sensome revealed by direct RNA sequencing." Nat Neurosci 16(12): 1896-1905.

Hong, S., et al. (2016). "New insights on the role of microglia in synaptic pruning in health and disease." Curr Opin Neurobiol 36: 128-134.

Hsiao, E. Y., et al. (2012). "Modeling an autism risk factor in mice leads to permanent immune dysregulation." Proc Natl Acad Sci U S A 109(31): 12776-12781.

Hsiao, E. Y., et al. (2013). "Microbiota modulate behavioral and physiological abnormalities associated with neurodevelopmental disorders." Cell 155(7): 1451-1463.

Hsueh, P. T., et al. (2017). "Expression of cerebral serotonin related to anxiety-like behaviors in C57BL/6 offspring induced by repeated subcutaneous prenatal exposure to low-dose lipopolysaccharide." PLoS One 12(6): e0179970.

Hui, C. W., et al. (2018). "Prenatal Immune Challenge in Mice Leads to Partly Sex-Dependent Behavioral, Microglial, and Molecular Abnormalities Associated with Schizophrenia." Front Mol Neurosci 11: 13.

Jones, K. L., et al. (2013). "Maternal diet rich in omega- 6 polyunsaturated fatty acids during gestation and lactation produces autistic-like sociability deficits in adult offspring." Behav Brain Res 238: 193199.

Juckel, G., et al. (2011). "Microglial activation in a neuroinflammational animal model of schizophrenia--a pilot study." Schizophr Res 131(1-3): 96-100.

Kaminska, B., et al. (2016). "Signal transduction and epigenetic mechanisms in the control of microglia activation during neuroinflammation." Biochim Biophys Acta 1862(3): 339-351.

Kim, S., et al. (2017). "Maternal gut bacteria promote neurodevelopmental abnormalities in mouse offspring." Nature 549(7673): 528-532.

Knuesel, l., et al. (2014). "Maternal immune activation and abnormal brain development across CNS disorders." Nat Rev Neurol 10(11): 643-660.

Krstic, D., et al. (2012). "Systemic immune challenges trigger and drive Alzheimer-like neuropathology in mice." J Neuroinflammation 9: 151.

Lai, A. Y., et al. (2013). "Distinct activation profiles in microglia of different ages: a systematic study in isolated embryonic to aged microglial cultures." Neuroscience 254: 185-195.

Lam, D., et al. (2017). "Responses of rat and mouse primary microglia to pro- and anti-inflammatory stimuli: molecular profiles, K(+) channels and migration." J Neuroinflammation 14(1): 166.

Le Belle, J. E., et al. (2014). "Maternal inflammation contributes to brain overgrowth and autismassociated behaviors through altered redox signaling in stem and progenitor cells." Stem Cell Reports 3(5): 725-734. 
Li, Q., et al. (2015). "Dietary supplementation with n-3 fatty acids from weaning limits brain biochemistry and behavioural changes elicited by prenatal exposure to maternal inflammation in the mouse model." Transl Psychiatry 5: e641.

Li, W. Y., et al. (2014). "Prenatal infection affects the neuronal architecture and cognitive function in adult mice." Dev Neurosci 36(5): 359-370.

Malkova, N. V., et al. (2012). "Maternal immune activation yields offspring displaying mouse versions of the three core symptoms of autism." Brain Behav Immun 26(4): 607-616.

Manitz, M. P., et al. (2013). "The role of microglia during life span in neuropsychiatric disease--an animal study." Schizophr Res 143(1): 221-222.

Manitz, M. P., et al. (2016). "Flow cytometric characterization of microglia in the offspring of Polyl:C treated mice." Brain Res 1636: 172-182.

Matcovitch-Natan, O., et al. (2016). "Microglia development follows a stepwise program to regulate brain homeostasis." Science 353(6301): aad8670.

Mattei, D., et al. (2014). "Minocycline rescues decrease in neurogenesis, increase in microglia cytokines and deficits in sensorimotor gating in an animal model of schizophrenia." Brain Behav Immun 38: 175-184.

Mattei, D., et al. (2017). "Maternal immune activation results in complex microglial transcriptome signature in the adult offspring that is reversed by minocycline treatment." Transl Psychiatry 7(5): e1120.

Meehan, C., et al. (2017). "Effects of immune activation during early or late gestation on schizophrenia-related behaviour in adult rat offspring." Brain Behav Immun 63: 8-20.

Meseguer, V., et al. (2014). "TRPA1 channels mediate acute neurogenic inflammation and pain produced by bacterial endotoxins." Nat Commun 5: 3125.

Meyer, U. (2014). "Prenatal poly(i:C) exposure and other developmental immune activation models in rodent systems." Biol Psychiatry 75(4): 307-315.

Meyer, U., et al. (2005). "Towards an immuno-precipitated neurodevelopmental animal model of schizophrenia." Neurosci Biobehav Rev 29(6): 913-947.

Meyer, U., et al. (2006). "The time of prenatal immune challenge determines the specificity of inflammation-mediated brain and behavioral pathology." J Neurosci 26(18): 4752-4762.

Meyer, U., et al. (2007). "The neurodevelopmental impact of prenatal infections at different times of pregnancy: the earlier the worse?" Neuroscientist 13(3): 241-256.

Missault, S., et al. (2014). "The risk for behavioural deficits is determined by the maternal immune response to prenatal immune challenge in a neurodevelopmental model." Brain Behav Immun 42: 138-146.

Monte, A. S., et al. (2017). "Two-hit model of schizophrenia induced by neonatal immune activation and peripubertal stress in rats: Study of sex differences and brain oxidative alterations." Behav Brain Res 331: 30-37. 
Morais, L. H., et al. (2018). "Strain differences in the susceptibility to the gut-brain axis and neurobehavioural alterations induced by maternal immune activation in mice." Behav Pharmacol.

Morgan, J. T., et al. (2010). "Microglial activation and increased microglial density observed in the dorsolateral prefrontal cortex in autism." Biol Psychiatry 68(4): 368-376.

Mortensen, P. B., et al. (2007). "Early infections of Toxoplasma gondii and the later development of schizophrenia." Schizophr Bull 33(3): 741-744.

Mosser, C. A., et al. (2017). "Microglia in CNS development: Shaping the brain for the future." Prog Neurobiol 149-150: 1-20.

Mosser, C. A., et al. (2017). "Microglia in CNS development: Shaping the brain for the future." Prog Neurobiol.

Mouihate, A. (2016). "Prenatal Activation of Toll-Like Receptor-4 Dampens Adult Hippocampal Neurogenesis in An IL-6 Dependent Manner." Front Cell Neurosci 10: 173.

Mukherjee, S. B. (2017). "Autism Spectrum Disorders - Diagnosis and Management." Indian J Pediatr 84(4): 307-314.

Nardone, S. and E. Elliott (2016). "The Interaction between the Immune System and Epigenetics in the Etiology of Autism Spectrum Disorders." Front Neurosci 10: 329.

Naviaux, R. K., et al. (2013). "Antipurinergic therapy corrects the autism-like features in the poly(IC) mouse model." PLoS One 8(3): e57380.

Nelson, L. H., et al. (2017). "Sex differences in microglial phagocytosis in the neonatal hippocampus." Brain Behav Immun 64: 11-22.

Nimmerjahn, A., et al. (2005). "Resting microglial cells are highly dynamic surveillants of brain parenchyma in vivo." Science 308(5726): 1314-1318.

Norden, D. M., et al. (2016). "Sequential activation of microglia and astrocyte cytokine expression precedes increased Iba-1 or GFAP immunoreactivity following systemic immune challenge." Glia 64(2): 300-316.

Notter, T., et al. (2017). "Translational evaluation of translocator protein as a marker of neuroinflammation in schizophrenia." Mol Psychiatry.

Notter, T., et al. (2018). "Reconceptualization of translocator protein as a biomarker of neuroinflammation in psychiatry." Mol Psychiatry 23(1): 36-47.

O'Loughlin, E., et al. (2017). "Acute in utero exposure to lipopolysaccharide induces inflammation in the pre- and postnatal brain and alters the glial cytoarchitecture in the developing amygdala." $\mathrm{J}$ Neuroinflammation 14(1): 212.

Paolicelli, R. C., et al. (2011). "Synaptic pruning by microglia is necessary for normal brain development." Science 333(6048): 1456-1458.

Paolicelli, R. C. and M. T. Ferretti (2017). "Function and Dysfunction of Microglia during Brain Development: Consequences for Synapses and Neural Circuits." Front Synaptic Neurosci 9: 9. 
Paylor, J. W., et al. (2016). "Developmental disruption of perineuronal nets in the medial prefrontal cortex after maternal immune activation." Sci Rep 6: 37580.

Pineda, E., et al. (2013). "Maternal immune activation promotes hippocampal kindling epileptogenesis in mice." Ann Neurol 74(1): 11-19.

Piontkewitz, Y., et al. (2011). "Abnormal trajectories of neurodevelopment and behavior following in utero insult in the rat." Biol Psychiatry 70(9): 842-851.

Pont-Lezica, L., et al. (2014). "Microglia shape corpus callosum axon tract fasciculation: functional impact of prenatal inflammation." Eur J Neurosci 39(10): 1551-1557.

Pratt, L., et al. (2013). "Maternal inflammation promotes fetal microglial activation and increased cholinergic expression in the fetal basal forebrain: role of interleukin-6." Pediatr Res 74(4): 393-401.

Ransohoff, R. M. and A. E. Cardona (2010). "The myeloid cells of the central nervous system parenchyma." Nature 468(7321): 253-262.

Reemst, K., et al. (2016). "The Indispensable Roles of Microglia and Astrocytes during Brain Development." Front Hum Neurosci 10: 566.

Reisinger, S., et al. (2015). "The poly(I:C)-induced maternal immune activation model in preclinical neuropsychiatric drug discovery." Pharmacol Ther 149: 213-226.

Ribeiro, B. M., et al. (2013). "Evidences for a progressive microglial activation and increase in iNOS expression in rats submitted to a neurodevelopmental model of schizophrenia: reversal by clozapine." Schizophr Res 151(1-3): 12-19.

Roumier, A., et al. (2008). "Prenatal activation of microglia induces delayed impairment of glutamatergic synaptic function." PLoS One 3(7): e2595.

Salter, M. W. and B. Stevens (2017). "Microglia emerge as central players in brain disease." Nat Med 23(9): 1018-1027.

Schaafsma, W., et al. (2017). "Maternal inflammation induces immune activation of fetal microglia and leads to disrupted microglia immune responses, behavior, and learning performance in adulthood." Neurobiol Dis 106: 291-300.

Schafer, D. P., et al. (2012). "Microglia sculpt postnatal neural circuits in an activity and complementdependent manner." Neuron 74(4): 691-705.

Schecter, R. W., et al. (2017). "Experience-Dependent Synaptic Plasticity in V1 Occurs without Microglial CX3CR1." J Neurosci 37(44): 10541-10553.

Schwartzer, J. J., et al. (2013). "Maternal immune activation and strain specific interactions in the development of autism-like behaviors in mice." Transl Psychiatry 3: e240.

Schwarz, J. M., et al. (2012). "Sex differences in microglial colonization of the developing rat brain." J Neurochem 120(6): 948-963.

Sekar, A., et al. (2016). "Schizophrenia risk from complex variation of complement component 4." Nature 530(7589): 177-183. 
Sipe, G. O., et al. (2016). "Microglial P2Y12 is necessary for synaptic plasticity in mouse visual cortex." Nat Commun 7: 10905.

Slusarczyk, J., et al. (2015). "Prenatal stress is a vulnerability factor for altered morphology and biological activity of microglia cells." Front Cell Neurosci 9: 82.

Smith, S. E., et al. (2007). "Maternal immune activation alters fetal brain development through interleukin-6." J Neurosci 27(40): 10695-10702.

Smolders, S., et al. (2015). "Maternal immune activation evoked by polyinosinic:polycytidylic acid does not evoke microglial cell activation in the embryo." Front Cell Neurosci 9: 301.

Solek, C. M., et al. (2017). "Maternal immune activation in neurodevelopmental disorders." Dev Dyn.

Sorensen, H. J., et al. (2009). "Association between prenatal exposure to bacterial infection and risk of schizophrenia." Schizophr Bull 35(3): 631-637.

Squarzoni, P., et al. (2014). "Microglia modulate wiring of the embryonic forebrain." Cell Rep 8(5): 1271-1279.

Streit, W. J., et al. (1999). "Reactive microgliosis." Prog Neurobiol 57(6): 563-581.

Streit, W. J., et al. (2014). "Microglial pathology." Acta Neuropathol Commun 2: 142.

Suvisaari, J., et al. (1999). "Association between prenatal exposure to poliovirus infection and adult schizophrenia." Am J Psychiatry 156(7): 1100-1102.

Swinnen, N., et al. (2013). "Complex invasion pattern of the cerebral cortex bymicroglial cells during development of the mouse embryo." Glia 61(2): 150-163.

Taylor, P. V., et al. (2012). "Sexually dimorphic effects of a prenatal immune challenge on social play and vasopressin expression in juvenile rats." Biol Sex Differ 3(1): 15.

Thion, M. S., et al. (2018). "Microbiome Influences Prenatal and Adult Microglia in a Sex-Specific Manner." Cell 172(3): 500-516 e516.

Torrey, E. F., et al. (1988). "Schizophrenic births and viral diseases in two states." Schizophr Res 1(1): 73-77.

Trepanier, M. O., et al. (2016). "Postmortem evidence of cerebral inflammation in schizophrenia: a systematic review." Mol Psychiatry 21(8): 1009-1026.

Tronel, C., et al. (2017). "Molecular Targets for PET Imaging of Activated Microglia: The Current Situation and Future Expectations." Int J Mol Sci 18(4).

Tronnes, A. A., et al. (2016). "Effects of Lipopolysaccharide and Progesterone Exposures on Embryonic Cerebral Cortex Development in Mice." Reprod Sci 23(6): 771-778.

Van den Eynde, K., et al. (2014). "Hypolocomotive behaviour associated with increased microglia in a prenatal immune activation model with relevance to schizophrenia." Behav Brain Res 258: 179-186.

van Kesteren, C. F., et al. (2017). "Immune involvement in the pathogenesis of schizophrenia: a metaanalysis on postmortem brain studies." Transl Psychiatry 7(3): e1075. 
VanRyzin, J. W., et al. (2016). "Temporary Depletion of Microglia during the Early Postnatal Period Induces Lasting Sex-Dependent and Sex-Independent Effects on Behavior in Rats." eNeuro 3(6).

Vargas, D. L., et al. (2005). "Neuroglial activation and neuroinflammation in the brain of patients with autism." Ann Neurol 57(1): 67-81.

Vezzani, A. and B. Viviani (2015). "Neuromodulatory properties of inflammatory cytokines and their impact on neuronal excitability." Neuropharmacology 96(Pt A): 70-82.

Weinhard, L., et al. (2017). "Sexual dimorphism of microglia and synapses during mouse postnatal development." Dev Neurobiol.

Weiser, M. J., et al. (2016). "Dietary docosahexaenoic acid alleviates autistic-like behaviors resulting from maternal immune activation in mice." Prostaglandins Leukot Essent Fatty Acids 106: 27-37.

Weiser, M. J., et al. (2015). "Dietary DHA during development affects depression-like behaviors and biomarkers that emerge after puberty in adolescent rats." J Lipid Res 56(1): 151-166.

Willi, R., et al. (2013). "Altered GSK3beta signaling in an infection-based mouse model of developmental neuropsychiatric disease." Neuropharmacology 73: 56-65.

Williamson, L. L., et al. (2012). "Environmental enrichment alters glial antigen expression and neuroimmune function in the adult rat hippocampus." Brain Behav Immun 26(3): 500-510.

Wu, W. L., et al. (2017). "The placental interleukin-6 signaling controls fetal brain development and behavior." Brain Behav Immun 62: 11-23.

Wu, Y., et al. (2015). "Microglia: Dynamic Mediators of Synapse Development and Plasticity." Trends Immunol 36(10): 605-613.

Xu, H., et al. (2016). "Environmental Enrichment Potently Prevents Microglia-Mediated Neuroinflammation by Human Amyloid beta-Protein Oligomers." J Neurosci 36(35): 9041-9056.

Xuan, I. C. and D. R. Hampson (2014). "Gender-dependent effects of maternal immune activation on the behavior of mouse offspring." PLoS One 9(8): e104433.

Yang, T. T., et al. (2013). "Differential distribution and activation of microglia in the brain of male C57BL/6J mice." Brain Struct Funct 218(4): 1051-1060.

Yanguas-Casas, N., et al. (2018). "Sex differences in the phagocytic and migratory activity of microglia and their impairment by palmitic acid." Glia 66(3): 522-537.

Zhan, Y., et al. (2014). "Deficient neuron-microglia signaling results in impaired functional brain connectivity and social behavior." Nat Neurosci 17(3): 400-406.

Zhu, F., et al. (2014). "Minocycline alleviates behavioral deficits and inhibits microglial activation in the offspring of pregnant mice after administration of polyriboinosinic-polyribocytidilic acid." Psychiatry Res 219(3): 680-686.

Ziv, Y., et al. (2006). "Immune cells contribute to the maintenance of neurogenesis and spatial learning abilities in adulthood." Nat Neurosci 9(2): 268-275. 